\title{
A comprehensive temporal patterning gene network in Drosophila medulla neuroblasts revealed by single-cell RNA sequencing
}

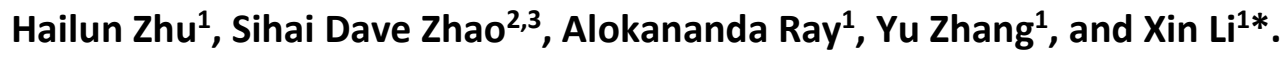

1. Department of Cell and Developmental Biology, University of Illinois at Urbana-Champaign, Urbana, IL 61801

2. Department of Statistics, University of Illinois at Urbana-Champaign, Urbana, IL 61801

3. Carl R. Woese Institute for Genomic Biology, University of Illinois at Urbana-Champaign, Urbana, IL 61801

(*) Corresponding author, email: lixin@illinois.edu 


\section{Abstract}

During development, neural stem cells are temporally patterned to sequentially generate a variety of neural types before exiting the cell cycle. Temporal patterning is well-studied in Drosophila, where neural stem cells called neuroblasts sequentially express cascades of Temporal Transcription Factors (TTFs) to control the birth-order dependent neural specification. However, currently known TTFs were mostly identified through candidate approaches and may not be complete. In addition, many fundamental questions remain concerning the TTF cascade initiation, progression, and termination. It is also not known why temporal progression only happens in neuroblasts but not in their differentiated progeny. In this work, we performed single-cell RNA sequencing of Drosophila medulla neuroblasts of all ages to study the temporal patterning process with single-cell resolution. Our scRNA-seq data revealed that sets of genes involved in different biological processes show high to low or low to high gradients as neuroblasts age. We also identified a list of novel TTFs, and experimentally characterized their roles in the temporal progression and neural fate specification. Our study revealed a comprehensive temporal gene network that patterns medulla neuroblasts from start to end. Furthermore, we found that the progression and termination of this temporal cascade also require transcription factors differentially expressed along the differentiation axis (neuroblasts $->->$ neurons). Lola proteins function as a speed modulator of temporal progression in neuroblasts; while Nerfin-1, a factor required to suppress de-differentiation in post-mitotic neurons, acts at the final temporal stage together with the last TTF of the cascade, to promote the switch to gliogenesis and the cell cycle exit. Our comprehensive study of the medulla neuroblast temporal cascade illustrated mechanisms that might be conserved in the temporal patterning of neural stem cells. 


\section{Introduction}

The heterogeneity of neural fates builds the foundation for constructing complex neural circuits. Integration of spatial and temporal patterning of neural stem cells allows neural progeny to adopt a spectrum of identities [reviewed in ${ }^{1-4}$ ]. Spatial patterning specifies distinct lineages, while within a specific lineage, temporal patterning further expands the neural diversity, as neural stem cells undergo gradual transitions along with self-renewal and give rise to a successive series of neural fates. A molecular mechanism of temporal patterning was identified in the nervous system of Drosophila. In Drosophila, neural stem cells called neuroblasts (NBs), were found to sequentially express certain cascades of temporal transcription factors (TTFs) and other temporal regulators that specify neural fates [reviewed in ${ }^{5,6}$ ]. The first TTF cascade was identified in the embryonic ventral nerve cord, where Hunchback (Hb), Kruppel (Kr), Nubbin/Pdm2 (Pdm), Castor (Cas) and Grainy head (Grh) are sequentially expressed in NBs as they age, and are required for the sequential specification of different neural fates ${ }^{7-12}$. Postembryonic NBs, including larval ventral nerve cord, central brain and optic lobe NBs, also employ temporal cascades that are yet different from the embryonic one ${ }^{13-20}$. In addition, the intermediate neural progenitors (INPs) of type II NBs are also temporally patterned by a TTF cascade to further expand the neural diversity ${ }^{21-23}$. In vertebrates, there is also accumulating evidence that neural stem cells undergo TTF dependent temporal patterning [reviewed in ${ }^{24,25}$ ]. For example, a number of transcription factors were shown to function in retinal progenitors or cortical progenitors to regulate temporal specification of neural fates ${ }^{26-35}$. Recently, single-cell transcriptomics studies of retinal progenitors and cortical progenitors revealed age-dependent dynamic changes in 
transcriptional profiles that are transmitted to their progeny ${ }^{28,36}$. These studies together suggest that TTF-dependent temporal patterning might be a general mechanism.

The model system we use to study temporal patterning is the medulla part of the Drosophila optic lobe. During development, a wave of neurogenesis sweeps from medial to lateral in the outer proliferation center (OPC) and sequentially converts symmetrically-dividing neuroepithelial cells (NE) into medulla NBs ${ }^{37-39}$. Medulla NBs divide asymmetrically multiple times to make a series of Ganglion Mother Cells (GMCs) which then divide to produce postmitotic progeny. Due to the spreading of the neurogenesis wave, neuroblasts of different ages and their progeny are orderly aligned on the medial to lateral spatial axis in the developing larval brain (Figure 1A). This feature makes medulla a great system to study temporal patterning. Previous studies showed that medulla NBs sequentially express Homothorax (Hth), Kumpfuss (Klu), Eyeless (Ey), Sloppy paired (SIp), Dichaete (D) and Tailless (TII) as they age ${ }^{18,19}$. Among them, Hth, Ey, SIp and D are each required for the expression of the corresponding neuronal transcription factors to control neural fates, but loss of Klu caused a NB proliferation defect, precluding examination of neural fates ${ }^{18,19,40}$. Similar to vertebrate retinal and cortical progenitors, medulla NBs switch to gliogenesis at the end of the lineage and then exit the cell cycle ${ }^{18,41}$.

In the medulla TTF cascade, there are still fundamental questions remaining. First, the several TTFs identified through candidate antibody screening may not compose the complete TTF sequence. Second, while Ey, SIp and D are each required for the next TTF to be activated, no cross-regulation was identified among Hth, Klu and Ey. Thus, it is not known how the Ey->SIp->D->TII TTF cascade is initiated. Third, it was not clear how the oldest medulla NBs switch to gliogenesis, end the temporal progression and exit the cell cycle. Finally, an even broader 
question concerns the regulation of the temporal cascade. As a previous TTF is only necessary but not sufficient to activate the next TTF in medulla NBs, additional regulators and molecular mechanisms might be involved to achieve the proper regulation of the cascade. Furthermore, temporal cascade progression only happens in neuroblasts but not in their differentiated progeny, which makes us to question whether genes functioning in the differentiation axis (NBs -> GMCs -> neurons) might play a role in regulating the temporal progression, which has never been studied before.

To discover all unknown TTFs and additional regulators, as well as to get a global view of the dynamic temporal patterning process of medulla neuroblasts, we applied single cell RNA sequencing (scRNA-seq) to our model system. ScRNA-seq is increasingly used as an unbiased approach to characterize heterogeneous tissues, including invertebrate and vertebrate nervous systems [reviewed in ${ }^{42-45}$ ]. The Drosophila medulla represents a great system to study temporal patterning using scRNA-seq, because at a single time point during development, we can obtain a continuous population of neuroblasts of all ages, and furthermore, we can use known TTFs to mark the neuroblast age and verify the inferred pseudotime trajectory. Applying scRNA-seq to the Drosophila medulla neuroblasts enabled us to capture all temporal stages and to reveal the gradual changing of neuroblast transcriptome with single-cell-cycle resolution. We observed growth and metabolism related genes showed high to low gradients, while genes involved in gene expression regulation and neural differentiation showed low to high gradients of expression as neuroblasts age. We identified a list of novel TTF candidates, among which, SoxNeuro (SoxN), doublesex-Mab related 99B (Dmrt99B), Odd paired (Opa), Earmuff (Erm), Scarecrow (Scro), BarH1, BarH2 and Glial cells missing $(\mathrm{Gcm})$ were confirmed to be required for the normal 
temporal patterning of medulla neuroblasts. We identified extensive cross-regulations among these novel TTFs and known TTFs, that generally follow the rule that a TTF is required to activate the next TTF and repress the previous TTF, but with important exceptions and complexities. Our study revealed a comprehensive temporal patterning cascade: Hth + SoxN + dmrt99B -> Opa -> $\mathrm{Ey}+\mathrm{Erm} \rightarrow \mathrm{Ey}+\mathrm{Opa}->\mathrm{SIp}+\mathrm{Scro} \rightarrow \mathrm{D} \rightarrow \mathrm{B}-\mathrm{H} 1 \& 2->\mathrm{TII}, \mathrm{Gcm}$, that controls the sequential generation of different neural types by regulating the expression of specific neuronal transcription factors. Finally, Gcm but not TIl is required for both the transition from neurogenesis to gliogenesis and the cell cycle exit. Moreover, in pursuit of the mechanism behind the regulation of the temporal cascade, we found that the progression and termination of the TTF temporal cascade require genes differentially expressed along the differentiation axis (NBs -> GMCs -> neurons) including Iola and nerfin-1, providing clues as to why the temporal progression only proceeds in neural stem cells.

\section{Results}

\section{ScRNA-seq revealed the dynamic transcriptome of Drosophila medulla neuroblasts as they age.}

To perform single-cell transcriptional profiling of Drosophila medulla neuroblasts, we dissociated dissected larval brains into single cell suspension and used fluorescence-activated cell sorting (FACS) to enrich medulla neuroblasts (Figure 1B). Medulla neuroblasts were sorted by the co-expression of two transgenes, SoxNGal4 driving UASRed that is expressed in all medulla cells, and $\mathrm{E}(\mathrm{spl}) \mathrm{my}::$ GFP that is expressed in all neuroblasts. We performed two rounds of scRNA-seq of sorted medulla neuroblasts using the 10x Genomics Chromium platform. We combined data from both sequencing experiments into a single analysis by integrating them using Seurat ${ }^{46}$. After 
quality control and filtering for neuroblasts, our data contained 3074 cells expressing between 261 and 6409 genes, with a median of 3682 expressed genes per cell.

To characterize the developmental states of sequenced neuroblasts, we used Seurat to partition the cells into 15 clusters, which we visualized on two-dimensional uniform manifold approximation and projection (UMAP) ${ }^{47}$ plots (Figure 1C). Among them, clusters 8, 11, 12, and 14 appear to be outliers, while all other clusters form a continuous cell stream, representing the main body of medulla neuroblasts. We also used Seurat to estimate the cell cycle phase of each cell (Figure 1D), based on the expression of known Drosophila cell cycle genes from Tinyatlas at Github (Supplementary Table 1). In the main body of medulla neuroblasts, cluster 13 contains mostly G1 cells, while cluster 2 contains mostly G2/M cells, and in other clusters cells of different phases are mixed. Next we inferred single-cell pseudotime trajectories using Monocle3 ${ }^{48-50}$ (Figure 1E). For this analysis, we removed the outlying clusters $8,11,12$, and 14 . The inferred developmental pseudotime appears to progress from right to left. Finally, the expression patterns of known TTFs in the medulla NB temporal cascade, in the order of Hth, Ey, SIp1/SIp2, D and TII, validated the inferred pseudotime trajectory of neuroblasts (Figure 1F). These data confirmed that our scRNAseq captured a continuous population of neuroblasts of all ages that formed a chronotopic map, with cluster 13 representing the newly-born NBs at G1 phase, cluster 0 containing the youngest neuroblasts that have started proliferation, and cluster 2 containing the oldest neuroblasts undergoing the terminal division (mostly at G2/M phases).

Two sets of genes show opposite expression gradients as medulla NBs age. 
Among the genes whose expression change with pseudotime, there are two sets of genes with high to low or low to high gradients, respectively. Genes encoding ribosomal proteins and metabolism enzymes showed significant high to low gradients, while genes involved in gene expression regulation and neural development showed low to high gradients. Negative regulators of cell growth and proliferation are gradually upregulated at later stages (Figure S1A,B) These data suggest that as medulla NBs age, the cell growth is gradually downregulated, while differentiation related genes are up-regulated.

\section{DEG analysis identified Candidate novel TTFs}

With the neuroblast transcriptome profile of all ages, we sought to identify more potential TTFs that had temporal expression patterns. Known TTFs were used to mark the relative age of a neuroblast, and indicate the position where a potential TTF might stand in the temporal cascade. We obtained a pre-compiled list of 755 Drosophila $\mathrm{TFs}^{51}$ identified based on bioinformatic analysis and manual curation. For each non-outlying cell cluster, we performed the DEG (Differentially Expressed Genes) analysis and identified the top 10 TFs in this list that were differentially expressed between the clusters (Figure S1C). We next examined the expression of these TFs at the protein level by immunostaining of $3^{\text {rd }}$ instar larval brains using available antibodies or GFP-fusion lines. To determine whether these TFs play crucial roles in the medulla temporal cascade, we examined cross-regulations between the novel TFs and known TTFs. We also assessed whether these TFs are involved in progeny fate specification. After screening through these TFs, we identified SoxN, Dmrt99B, Opa, Erm, Scro, BarH1, BarH2 and Gcm as novel TTFs in the temporal cascade (Figure 1G, H). According to the scRNA-seq data, SoxN transcripts 
are already present in the newly-born NBs similar to Hth, remain high in the youngest NBs, and then become reduced from the Ey stage to the D stage and increase again after the D stage; Dmrt99B transcripts are present from the newly-born NBs until the Slp stage NBs; the expression of Opa transcripts starts in the youngest NBs before Ey, and continues until the end of the Ey stage, but with a small gap in the middle corresponding to the early Ey stage; Erm transcripts are present at this gap between the two groups of opa-expressing NBs, i.e. in the early Ey stage NBs; Scro transcripts are present in NBs from about the Slp stage to the final stage; BarH1 and BarH2 transcripts are present in a group of NBs older than the D stage NBs but younger than the TII stage NBs; Gcm transcripts are present in the NBs of the final stage, later than the TIl stage (Figure 1G,H). For a few other candidate TFs with temporal expression patterns, including Oaz, Hbn, Scrt and Sba (Figure 1H), we either did not observe an effect on the temporal progression (Oaz, Sba, Scrt) using available RNAi lines, or we are lacking effective reagents ( $\mathrm{Hbn})$. Therefore, we did not include these TFs in further analysis.

SoxN and Dmrt99B cooperate with Hth to specify the first temporal identity. According to our scRNA-seq data, SoxN transcripts have a rather broad distribution in NBs. However, antibody staining showed that SoxN protein is only expressed before the Ey stage (Figure 2A- $\left.\mathrm{A}^{\prime \prime}\right)$, indicating that special post-transcriptional regulation of SoxN exists at later stages. SoxN is a SoxB family HMG-domain transcription factor involved in neuroblast formation and neuron differentiation in the ventral nerve $\operatorname{cord}^{52-55}$. Its expression has been noted in the medulla ${ }^{19}$, but its function in the medulla has not been studied. 
Similar to Hth, SoxN expression starts in NE cells before they are transformed into NBs marked by Deadpan (Dpn), and continues in the youngest NBs until the Ey stage (Figure 2A- $A^{\prime \prime}$ ). SoxN is also inherited in GMCs and neurons generated before the Ey stage (Figure S2A, $A^{\prime}$ ). To test if SoxN is required for neuron fate specification, we generated SoxN homozygous mutant clones in otherwise heterozygous brains using the MARCM system ${ }^{56}$. First we observed that SoxN staining is lost in SoxN mutant clones, validating the specificity of the antibody ${ }^{55}$ and the mutant (Figure S2B, $\mathrm{B}^{\prime}$ ). In the mutant clones, Bsh (brain-specific homeobox) expressing neurons which are generated in the $\mathrm{Hth}$ stage ${ }^{18,19,57}$ were lost, suggesting that SoxN cooperates with $\mathrm{Hth}$ to specify the Bsh neuron fate (Figure 2B-B"). Moreover, with loss of SoxN, Hth expression in NBs was not affected, and the following temporal cascade still proceeded as indicated by the Ey and SIp expression in mutant NBs (Figure S2C- $D^{\prime \prime \prime}$ ). These results suggest that SoxN is not required for the initiation of the temporal gene cascade in NBs. To test if the termination of SoxN is due to the cascade progression, we generated ey RNAi clones using the $a y G a l 4$ system ${ }^{58}$ as well as s/p mutant MARCM clones. SoxN expression was expanded into older NBs in ey RNAi clones but not affected in slp mutant clones, suggesting that Ey inhibits the expression of SoxN (Figure 2C-C", Figure S2E-E"). Therefore, SoxN is another TTF that determines the first temporal stage together with Hth. However, neither SoxN nor Hth is required for the progression of the cascade.

Another TF gene expressed in the youngest NBs is dmrt99B, encoding a DMRT (doublesexand mab-3-related) transcription factor ${ }^{59,60}$. A Dmrt99B::GFP fusion protein expressed under genomic BAC endogenous control (from modERN Project) is turned on before the NE to NB transition and remains high in young NBs, and decreases at the beginning of the Slp stage (Figure 2D-D"). In dmrt99B RNAi clones generated using the ayGal4 system, the NE to NB transition is 
not affected, but the Bsh expression is lost (Figure 2E-E', Figure S2G, G'), suggesting that Dmrt99B is also required for the first temporal fate. In contrast to SoxN and Hth, Dmrt99B is required for the later TTF cascade. In dmrt99B RNAi clones, the expression of the next TTF Opa (see the section below) is lost or dramatically delayed (Figure $2 \mathrm{~F}, \mathrm{~F}^{\prime}$ ), SIp2 is also delayed and D is lost (Figure S2H$\left.H^{\prime \prime \prime}\right)$.

In summary, SoxN, Dmrt99B and Hth are three TTFs that are first turned on in the NE and all of them are required for the first temporal fate, but only loss of Dmrt99B affected the proper progression of the subsequent temporal cascade (Figure $3 \mathrm{~L}$ ). However, it is possible that partial redundancy may exist within these three TTFs.

\section{Opa is the missing link between the NE TTFs and the NB TTF cascade.}

Opa is a zinc finger transcription factor homologous to mammalian Zic proteins, and has been shown to function in the embryonic patterning process as a pioneer factor ${ }^{61,62}$, as well as in adult head development ${ }^{63,64}$. In the type II neuroblast lineage, Opa is expressed in early-born INPs and required for the temporal progression of the INP temporal cascade ${ }^{23}$.

We examined Opa protein expression in the medulla, and observed that it is expressed in two stripes of NBs, consistent with the transcript pattern (Figure 3A- $A^{\prime \prime}$ ). Opa is not expressed in NE cells, and its expression is activated at the same time as Dpn in the youngest NBs (Figure 3A$\left.A^{\prime \prime}\right)$. The first Opa stripe is more towards the lateral compared to the Ey stripe (i.e. the first wave of Opa expression in NBs is before Ey), while the second stripe is overlapping with the medial half of the Ey stripe (i.e. the second wave of Opa expression is in late Ey stage NBs) (Figure 3B, B'). The second stripe of Opa expression terminates as Slp expression reaches its peak (Figure 3C, $C^{\prime}$ ). 
Inside the medulla, two layers of Opa-expressing neurons, seemingly born from the two Opa windows, are observed. The neurons eventually stop expressing Opa during maturation (Figure S3A-A").

To test whether Opa is required in the temporal cascade, we generated opa mutant clones. With loss of Opa, Ey expression and the subsequent temporal cascade, as indicated by SIp2 expression, are greatly delayed (Figure S3B-B"'). The reason for a delay but not a complete loss of Ey expression might be that the clones are not generated early enough to completely eliminate Opa expression. To circumvent this problem, we used vsxG4, which is expressed in the central compartment of the medulla crescent (cOPC) ${ }^{65}$ (Figure $\left.\mathrm{S} 3 \mathrm{~L}\right)$, to drive opa RNAi eliminating Opa expression from the start. In the region where Opa expression was absent, Ey expression and SIp2 expression were mostly lost (Figure 3D- $\left.D^{\prime \prime}\right)$, suggesting that Opa is necessary for the temporal cascade to normally proceed towards the Ey stage. We also used another regional Gal4, optixG4, which is expressed in the main arms of the medulla crescent (mOPC regions) (Erclik et al., 2017) (Figure S3L) to drive opa RNAi and obtained the same phenotype. Meanwhile, in the region with opa $\mathrm{RNAi}$, Hth expression was expanded in both NBs and progeny (Figure 3E, F, $\mathrm{F}^{\prime}$ ). Therefore, Opa is required to repress the previous TTF Hth, and activate the next TTF Ey.

Next, we examined whether known TTFs regulate opa expression. In ey RNAi clones, Opa expression persisted without a gap from the youngest NBs to the oldest NBs (Figure $3 G, G^{\prime}$ ), suggesting that Ey (and/or any later TTF in the temporal cascade) is required to repress Opa in both the gap and the older NBs. In addition, neurons born at different time points all inherit Opa in the ey RNAi clones. Since the termination of the second stripe of Opa corelates with the upregulation of SIp, and Ey is required to activate SIp, it is possible that the de-repression of Opa 
in older NBs in ey RNAi clones is due to loss of Slp. To test whether Slp is required to terminate the second wave of Opa expression, we generated slp mutant clones, inside which the expression of the second stripe of Opa expanded towards the oldest NBs (Figure $3 \mathrm{H}, \mathrm{H}^{\prime}$ ). However, the gap between the two stripes was not affected in slp mutant clones.

Finally, we examined whether Opa controls the specification of neural fates. A layer of neurons expressing Runt are born between the Hth stage and the Ey stage ${ }^{19}$ (Figure S3C). Runt expressing neurons are unaffected in hth mutant clones, and they are expanded in ey mutant clones, suggesting that a TTF repressed by Ey is responsible for the generation of Runt neurons

(Figure S3D, E, E'). Consistently, over-expression of Ey in NBs is sufficient to block the production of Runt neurons (data not shown). Klu was implicated as a TTF specifying Runt neurons because over-expression of Klu led to extra Runt neurons ${ }^{19}$. However, Klu and Ey do not regulate the expression of each other, and klu mutant caused ectopic NB phenotype, precluding examination of neural fates ${ }^{19}$. Since the first stripe of Opa is turned on after Hth and before Ey, and we have shown that Ey is required to repress Opa, we examined whether Opa is required to specify Runt neuron fate. In opa RNAi regions, Runt neurons are largely lost (Figure 3F,F"), suggesting that the first stripe of Opa indeed serves as a TTF between the Hth stage and the Ey stage, and is required for Runt neuron determination (Figure $3 \mathrm{~L}$ ).

\section{Erm is required to generate the two-stripe expression pattern of Opa}

According to the scRNA-seq data, Erm transcripts are present at the gap between the two groups of Opa-expressing NBs. Erm, an ortholog of mammalian Fezf2, is a zinc-finger transcription factor shown to be expressed in INPs of type II neuroblast lineages where it maintains the INPs' 
restricted developmental potential ${ }^{66-68}$. A role for Erm in temporal patterning has not been studied before. We used an Erm::V5 line that recapitulates the true expression of Erm ${ }^{69}$ to examine the protein expression pattern of Erm in the medulla. The staining for V5 marker showed that Erm is indeed expressed at the gap between the two Opa-expressing stripes in NBs, consistent with the mRNA pattern (Figure 3I-I"). In addition, Erm is also inherited in the progeny located between the two layers of $\mathrm{Opa}^{+}$neurons (Figure S3F). Interestingly, there is another stripe of Erm expressed in NE cells adjacent to the youngest NBs (Figure 3I-I"). The expression pattern of Erm in NBs suggests that it might repress Opa expression and generate the gap. To address this hypothesis, we generated $\mathrm{erm}^{1}$ mutant clones, and showed that with loss of Erm, the gap in Opa expression in NBs and progeny was no longer present (Figure 3J,J', Figure S3G,G'), suggesting that Erm does function to repress Opa at the gap, possibly through cooperation with Ey. In erm mutant clones, Ey expression became weaker but was still present, and Hth expression was expanded (Figure S3H-I'). In erm RNAi clones, Opa showed the same de-repression phenotype (data not shown). In summary, we showed that Erm is activated in NBs at a similar time as Ey, and is required to repress Opa to generate the gap in Opa expression.

\section{The Ey stage is divided into two sub-temporal stages by Erm and the second stripe of Opa}

The expression patterns and cross-regulations between Erm, Opa and Ey suggest that the

Ey stage can be subdivided into two sub-temporal stages, with early Ey stage NBs co-expressing Ey and Erm, and late Ey stage NBs co-expressing Ey and the second stripe of Opa (Figure 1F,G,H, Figure $\left.3 B, B^{\prime}, I, I^{\prime}\right)$. It is possible that Ey stage NBs generate different neural types in these two subtemporal stages. To test this hypothesis, we set out to examine whether loss of erm or opa affects 
the Ey progeny fates. As previously reported ${ }^{18}$, each medulla GMC divides to generate a Notchon neuron which expresses Apterous (Ap) and a Notch-off neuron which doesn't express Ap. The Notch-off neurons generated in the Ey stage inherit Ey expression, and they also express a bHLH transcription factor Knot (Kn) (Figure S3J-J"'). Ey is required for Kn expression, because Kn is lost in ey RNAi clones (Figure $\mathrm{S} 3 \mathrm{~K}, \mathrm{~K}^{\prime}$ ). Drifter (Dfr, also known as $\mathrm{Vvl}$ ) is another transcription factor expressed in the Ey stage progeny, and is lost in ey mutant or RNAi clones ${ }^{18,19}$. Dfr expressing neurons can be divided into two large populations. The first population is a layer of Notch-on neurons generated in the early Ey stage that express both Dfr and Ap (Figure S3 M-N', Dfr' cells between the two white dashed lines), and among this population some neurons also express a weak level of Dac, thus expressing all three TFs (white arrows in Figure S3 M-N'). The second population includes clusters of later-born Notch-off neurons that express both Dfr and Dac, but not $A p^{18,19,57}$ (Figure $S 3 M^{\prime}, N^{\prime}$, purple cells enclosed by green dashed lines). The $\mathrm{Dfr}^{+}$Notch-on neurons are specified into several neural types including Tm3, Tm9, Mi10 etc ${ }^{57}$. The $\mathrm{Dfr}^{+} \mathrm{Dac}^{+}$ Notch-off neurons are only generated in certain spatial domains, and they are specified as Dm8 multi-columnar neurons 57,70 . These $\mathrm{Dfr}^{+} \mathrm{Dac}^{+} \mathrm{N}$-off neurons are still produced in slp mutant clones, suggesting that they are born before the SIp stage (Figure S3O,O').

Using these markers, we examined whether Erm is required for the specification of neural fates. In erm RNAi clones, Kn expressing neurons are always lost on the dorsal side of the medulla, but still present on the ventral side (Figure $3 \mathrm{~K}, \mathrm{~K}^{\prime}$ ); the first population of Dfr expressing neurons are largely lost, but the second population neurons expressing both Dfr and Dac generated at a later stage are still present (Figure $\left.3 \mathrm{M}, \mathrm{M}^{\prime}\right)$. In erm mutant clones, we observed the same phenotype (Figure $3 N, N^{\prime}$ ). These data suggest that Erm is required for the generation of the first 
population of $\mathrm{Dfr}^{+}$neurons in the early Ey stage but not for the second population (Figure $3 \mathrm{~L}$ ).

Erm is also required for the production of Kn expressing neurons in the dorsal medulla, but Kn expressing neurons are still present in the ventral medulla with loss of Erm, and a possible reason is that another population of $\mathrm{Kn}^{+}$neurons not dependent on Erm are generated in the ventral medulla only, possibly in the late Ey stage.

Next we examined the expression of $\mathrm{Kn}$, Dfr and Dac in opa RNAi clones. Since Opa is required for Ey expression, and Ey is required for Kn and Dfr expression, we would expect that Kn and Dfr are lost in opa RNAi clones. However, only Kn is lost in opa RNAi clones (Figure S3P,P'), while Dfr expression is not lost and even expanded, suggesting that Dfr expression does not require Ey if Opa is not present. However, neurons expressing both Dfr and Dac are lost in opa RNAi clones (Figure 30-O"). These together suggest that Opa normally represses the generation of the first population $\mathrm{Dfr}^{+}$neurons ( $\mathrm{Dfr}^{+} \mathrm{Ap}{ }^{+}$neurons), but is required for the generation of $\mathrm{Dfr}^{+}$ $\mathrm{Dac}^{+}$neurons (second population). Erm and Ey together are required to turn off Opa at the early Ey stage to allow for the generation of $\mathrm{Dfr}^{+} \mathrm{Ap}^{+}$neurons. After Erm is turned off, the second stripe of Opa together with Ey then promote the generation of $\mathrm{Dfr}^{+} \mathrm{Dac}^{+}$Notch-off neurons, possibly acting together with spatial factors. In summary, our data showed that the Ey stage is divided into (at least) two sub-temporal stages with Ey/Erm, and Ey/Opa as TTFs, respectively, and these different combination of TTFs determine different neural fates. (Figure 3L)

Scro is required to promote Slp expression to the threshold level for temporal transition. 
Based on our scRNA-seq data, scro mRNA is expressed in NBs starting at a similar time as SIp 1 and 2. The scro gene encodes a NK-2 homeobox transcription factor, the expression of which in the medulla has been indicated by its knock-in mutant alleles in a recent study ${ }^{71,72}$.

To study the function of Scro in the temporal cascade, we generated scro RNAi clones, and observed that in such clones, Slp expression was greatly reduced (Figure 4A, $A^{\prime}$ ), while Opa and Ey expression was expanded into older NBs, and D expression was lost (Figure 4B-D'). This set of data suggest that Scro promotes the transition from the Ey stage to the Slp stage by activating SIp expression. Since Slp is required to repress Ey and Opa, as well as to activate D, the expansion of Opa and Ey as well as the absence of D expression in scro RNAi clones might be due to the weak Slp expression caused by loss of Scro, but a direct role for Scro also cannot be excluded (Figure 4J). Next, we tested Scro's effect on the neuron fate generated in the SIp temporal window. Sox $102 \mathrm{~F}$ is a transcription factor expressed in subsets of neuronal progeny of the Slp stage and D stage NBs (Figure S4A), and it is lost in s/p mutant clones ${ }^{40}$. In scro RNAi clones, Sox $102 \mathrm{~F}^{+}$neurons were also largely lost, showing that Scro is required for the neuron fate generated in the Slp stage (Figure $4 \mathrm{E}, \mathrm{E}^{\prime}$ ). Thus our data suggest that Scro is required to activate SIp expression to the full level that allows the actual transition to the Slp stage to occur (Figure 4J), because with loss of Scro, although a weak level of Slp is still expressed in NBs, it is not sufficient to specify the correct neural type, or promote temporal progression of the cascade.

\section{BarH genes mark the temporal stage between the D stage and the Tll stage}

BarH1 and BarH2 are two homologous genes encoding homeobox transcription factors, best known for their function in the development of the eye $\mathrm{e}^{73,74}$. In the medulla, consistent with 
our scRNA-seq data, both genes are expressed in medulla NBs between the D stage and the TII stage. D, BarH proteins, and TII are expressed in three consecutive stripes with BarH proteins in the middle (Figure 4F-F", Figure S4B-B"'). While D expression and TIl expression do not overlap with each other, the expression of BarH1 and BarH2 overlaps with both D and TII. BarH1 and BarH2 are initiated at almost the same time, and their stripes in NBs are much overlapped, but the highest level of expression of the two TFs is achieved at different times in different cells (Figure 4G-G'). The inequivalence of their expression is amplified in neurons that are born at the BarH stage, as neurons expressing only BarH1 or $\mathrm{BarH} 2$ are observed inside the medulla (Figure S4C-C').

If BarH1 and BarH2 are TTFs between $\mathrm{D}$ and TIl, they might be activated by $\mathrm{D}$, and be responsible for terminating $D$ expression and activating TIl expression. We used ayG4 to drive $D$ RNAi, and observed loss of BarH1 expression in D-RNAi clones (Figure $4 \mathrm{H}, \mathrm{H}^{\prime}$ ), suggesting that $\mathrm{D}$ is required for $\mathrm{BarH} 1$ activation. To test whether $\mathrm{BarH} 1$ and $\mathrm{BarH} 2$ are required for the transitions from the D stage to the Tll stage, we generated clones in which RNAi of both BarH genes were induced. Inside such RNAi clones, TIl was lost while D was expanded to the oldest NBs (Figure 4II'). Next, we tested whether BarH1 and BarH2 are individually required in the transitions. With loss of BarH1 or BarH2 alone, normal Tll expression was observed in NBs (Figure S4D, F), suggesting that BarH1 and BarH2 act redundantly to activate the next TTF TII (Figure 4J).

\section{$\mathrm{Gcm}$ is the final TTF required for the switch to gliogenesis and cell cycle exit}

Previously it was thought that TII is the last TTF expressed in the oldest medulla neuroblasts that produce glia, but whether TII indeed plays a role in gliogenesis hasn't been 
examined $^{18}$. Our scRNA-seq data suggest that there is another temporal stage after the TII stage marked by the expression of Gcm and Dacapo (Dap) (Figure 1G, FigureS5A). Gcm, a zinc finger transcription factor, was shown to be essential for glial fate determination in the embryonic nervous system and larval visual system ${ }^{75-78}$. Then another study showed that Gcm is expressed in a group of precursors located at the border between the optic lobe and the central brain and required to generate medulla neuropil glia $(\mathrm{mng})^{41}$. Our scRNA-seq data suggest that these precursors are the final stage of medulla neuroblasts, rather than a separate group of dedicated glial precursors.

A Gcm::GFP-BAC line was used to examine the protein expression pattern of $\mathrm{Gcm}$, and it was confirmed that Gcm protein is expressed after Tll in the oldest medulla NBs (Figure 5A-A" and Figure S5B-B"). Gcm expression has a significant overlap with TII, but in a more restricted stripe closer to the central brain. A high level of $\mathrm{Gcm}$ expression is often observed in neuroblasts with a reduced level of TII. Gcm-expressing progeny later activate Repo expression, as suggested by the co-expression of Gcm::GFP and Repo in the migrating mng generated by the oldest medulla NBs (Figure S5C-C'). As mng migrate towards the medulla neuropil, Gcm expression reduces, while the expression of $\mathrm{Gcm} 2$, a homolog of $\mathrm{Gcm}$, increases (Figure S5D-D"). Gcm2 is not expressed in the neuroblasts ${ }^{41}$ (Figure S5D-D'). Although some Gcm-expressing NBs and newly generated mng still transiently retain a low level of TII, most TII-expressing progeny do not express Gcm or Repo, instead, some of them express Dac (Figure S5E-E"), suggesting that TII is a TTF activated before $\mathrm{Gcm}$, and that TIl stage NBs produce $\mathrm{TII}^{+}$neurons including some expressing both TII and Dac. These neurons appear to only express TII transiently, and TII is turned off in mature neurons. 
Thus, Gcm is expressed at the final stage when NBs transit from neurogenesis to gliogenesis and subsequently exit the cell cycle. Dap, a cell cycle inhibitor orthologous to vertebrate Cdkn1a/P21, is expressed in a similar pattern as Gcm (Figure 1G, FigureS5A). TII is expressed in a slightly earlier stage, and loss of TIl does not affect the cell cycle exit or glia production (Figure S5F-F"). Thus, Gcm might be the critical regulator that functions in promoting gliogenesis and cell cycle exit.

We examined the function of $\mathrm{Gcm}$ by generating $\mathrm{gcm}$ mutant clones. In a wild-type brain, cells expressing both Dpn and TIl are not present deep inside the medulla where only neurons and glia are located. However, with loss of $\mathrm{gcm}$, ectopic $\mathrm{Tll}^{+} \mathrm{Dpn}^{+}$cells are observed at deeper planes (Figure 5B-B"). Those cells should be the $\mathrm{TII}^{+}$neuroblasts unable to transit into the glia producing and cell cycle exiting mode, and instead, they remain at the neuron producing mode and keep producing supernumerary $\mathrm{TII}^{+} \mathrm{Dac}^{+}$neurons (Figure 5C- $\mathrm{C}^{\prime \prime}$ ). In gcm RNAi clones, we also observed excessive $\mathrm{TII}^{+} \mathrm{Dac}^{+}$neurons (Figure S5G-G"). These results suggest that $\mathrm{Gcm}$ is required to repress $\mathrm{TII}$, and end the temporal progression. The extended NB proliferation could be due to the loss of Dap expression in gcm mutant clones (Figure 5D-D"). Confirming Gcm's known role in gliogenesis, we never observed $\mathrm{Repo}^{+}$glia around the medulla neuropil inside $\mathrm{gcm}$ mutant clones or gcm RNAi clones (Figure 5E-E'). To determine if $\mathrm{Gcm}$ is sufficient to promote gliogenesis and terminate neuroblast proliferation, we tested the effect of misexpression of Gcm in younger NBs. Clones mis-expressing Gcm were small, and composed mostly of neuroblasts marked by Dpn and glia marked by Repo, suggesting that $\mathrm{Gcm}$ is sufficient to promote gliogenesis, but may cooperate with other factors to promote cell cycle exit (Figure $\mathrm{S} 5 \mathrm{H}-\mathrm{H}^{\prime \prime}$ ). In summary, this set of data support 
that $\mathrm{Gcm}$ is the final TTF required for the switch to gliogenesis and for ending the temporal cascade by activating Dap.

As TIl is not required for glia production suggesting that TII is not required to activate $\mathrm{Gcm}$, there should be another TTF directly upstream of $\mathrm{Gcm}$ and required for $\mathrm{Gcm}$ expression. Therefore, we tested if BarH genes are the upstream TTFs. Because of the lack of Gcm antibody, we used Repo expression as an indicator for the successful progression towards the final temporal stage. While mng produced by the oldest NBs line up around the medulla neuropil continuously in a wild-type brain (Figure 5F-F"), in BarH1 and BarH2 double RNAi clones, mng was lost, suggesting that $\mathrm{BarH} 1$ and $\mathrm{BarH} 2$ are upstream of $\mathrm{Gcm}$ in the temporal cascade (Figure 5G-G'). However, mng production was normal with individual RNAi of BarH1 or BarH2, suggesting that $\mathrm{BarH} 1$ and $\mathrm{BarH} 2$ act redundantly to promote the temporal cascade towards the final stage (Figure S4E, G). In summary, BarH1 and BarH2 are required to activate both TIl and $\mathrm{Gcm}$, but with different kinetics, and $\mathrm{Gcm}$ is then required to promote gliogenesis and cell cycle exit by repressing TII and activating Dap (Figure $5 \mathrm{H}$ ).

\section{TFs functioning along the differentiation axis participate in the temporal cascade regulation.}

Our scRNA-seq analysis enabled us to identify a fairly complete temporal cascade from start to end. An important question concerning the regulation of the temporal cascade is why the progression of the TTF cascade is only present in NBs, but not in neurons. Our hypothesis is that factors differently expressed between NBs and neurons might contribute to limit the sequential activation of TTFs to NBs. To test this hypothesis, we screened through some TFs that are not expressed in a TTF manner but rather differentially expressed along the differentiation axis (NBs 
$\rightarrow$ GMCs $->$ neurons), and we found that two genes, longitudinals lacking (lola) and nervous

fingers 1 (nerfin-1), participate in the temporal cascade regulation.

The gene lola encodes about 20 isoforms of transcription factors belonging to a Broadcomplex, Tramtrack and Bric-à-brac/poxvirus and zinc finger (BTB/POZ) family of proteins. The isoforms have a common BTB domain and different Zinc fingers that give each isoform different DNA binding specificities ${ }^{79}$. The 20 isoforms show different expression patterns in the medulla. For example, Lola-F (isoform nomenclature as in $\operatorname{Ref}^{79}$ ) is expressed in all NBs, all GMCs and newly born neurons, but is down-regulated quickly to absence as neurons mature (Figure 6A-A", Figure S6A-A"). Lola- $\mathrm{N}$ is expressed mostly in mature neurons ${ }^{80}$. A Lola-T::GFP-BAC line showed weak Lola-T::GFP expression in a similar pattern as that of Lola-F, although with a much earlier activation starting from NE (Figure 6B, $\mathrm{B}^{\prime}$ ). A Lola-K::GFP-BAC line showed that Lola-K is expressed at a high level in mature neurons and NBs, but is not detected in GMCs (Figure 6C, $C^{\prime}$ ). Therefore, different isoforms of Lola could have diverse functions in NBs, GMCs and neurons. Overall, this change in Lola isoform composition happens along the differentiation axis. For example, only NBs express both Lola-F and Lola-K, which might act together to regulate NB-specific processes. Using optixG4 which is expressed in the $\mathrm{mOPC}^{65}$, or ayG4 to drive lola RNAi that eliminates all isoforms of Lola, we observed a great expansion of Hth expression in NBs, a slight expansion of the first stripe of Opa, and delays in Ey expression, the second stripe of Opa, and Slp expression to increasing extents in NBs (Figure 6D-G", Figure S6B-D"). The first stripe of Opa was only slightly expanded, but the second stripe of Opa is further delayed as the gap between the two stripes of Opa became much larger; the delay of Ey expression was very severe and the SIp expression was often absent. This is as if the temporal progression grows slower and slower (Figure 6P). With 
Iola RNAi driven by optixG4 or ayG4, we also observed a great reduction of Runt neurons and Kn neurons, suggesting that Lola proteins are also required for the neuronal fate specification (Figure $6 \mathrm{E}$ and data not shown). This set of data suggest that Lola inhibits Hth expression and facilitates the normal temporal progression, and Lola might be a speed modulator of the temporal cascade in NBs (Figure 6P).

Nerfin-1 is a zinc-finger transcription factor expressed in postmitotic neurons and is required for maintaining their differentiated status ${ }^{81,82}$. According to our scRNA-seq data, Nerfin1 transcripts are present in the oldest NBs similar to Gcm transcripts (Figure $1 G, H$ ). Using a Nerfin1::GFP-BAC line, we showed that Nerfin-1 protein is expressed mostly in maturing neurons as previously reported (Figure 6H). However, the co-expression of Dpn and Nerfin-1 can be observed in the nuclei of the oldest NBs (Figure 6H-I'). Nerfin-1 expression persists in the newborn glia generated by the oldest NBs for a short time and is lost as the glia mature and migrate, suggested by the fact that Repo seldom co-expresses with Nerfin-1 in the mng glia located around the neuropil (data not shown). Next, we tested whether Nerfin-1 is required for the cell cycle exit and glia production. We used eyG4 to drive nerfin-1 RNAi, and showed that Nerfin-1 is another critical regulator for the final-stage NBs. In a wild type control brain, mng produced from the oldest NBs line up the medulla neuropil continuously (Figure 6J). In contrast, with loss of Nerfin1, only a few scattered mng are observed around the medulla neuropil (Figure 6K)(the reduction is highly significant: $p=1.15 \times 10^{-10}$ by $t$-test, $n=5$ brains each). This suggests that the transition to gliogenesis is affected by the loss of Nerfin-1, and it is possible that NBs could be stuck at the previous TIl stage. With loss of Nerfin-1, we also observed numerous ectopic $\mathrm{Dpn}^{+}$neuroblasts inside the medulla (Figure 6M), consistent with previous reports showing that loss of Nerfin-1 
causes de-differentiation of neurons back into neuroblasts ${ }^{81,82}$. However, if the oldest NBs fail to exit the cell cycle, they can also be among these ectopic neuroblasts. To test this possibility, we examined TIl expression. TII is transiently expressed in the newly-born progeny from $\mathrm{TII}^{+} \mathrm{NBs}$, and will be lost soon after. In a wild type brain at a deeper focal plane, NBs are only observed at the surface, and TII is observed in old NBs and their newly-born progeny just below the surface (Figure $6 \mathrm{~N}$ arrows). The lineages in the middle of the brain have been completed (NBs have finished generating mng and exited the cell cycle), and thus no NB or $\mathrm{Tll}^{+}$progeny is observed (Figure $6 \mathrm{~N}$ ). However, with loss of Nerfin-1, $\mathrm{TII}^{+}$progeny continue to be produced throughout the brain (Figure 60). This set of data suggest that Nerfin-1 is also required for the transition from the TIl stage to gliogenesis and for exiting the cell cycle. Thus Nerfin-1, which is required in neurons to maintain their differentiation status, is turned on in the oldest NBs to promote gliogeneis and put a stop on the temporal progression (Figure 6Q).

Together, the requirement of Lola and Nerfin-1 in the normal cascade progression and termination suggests that genes that are differentially expressed and functioning along the differentiation axis also contribute to the temporal cascade regulation.

\section{Discussion}

\section{ScRNA-Seq is a powerful tool to study temporal patterning.}

Our scRNA-Seq analysis revealed the temporal progression of transcriptional profiles as medulla neuroblasts age at single-cell resolution. We discovered candidates of critical temporal patterning regulators including eight novel TTFs, as well as TFs such as Lola and Nerfin-1, which are expressed along the differentiation axis but also involved in the temporal patterning process. 
Further experimental validation of novel TTFs and other crucial regulators confirmed the accuracy of our high-resolution data, supporting that scRNA-seq is a powerful tool to study the highly dynamic temporal patterning process. Our analysis and further experimental investigation revealed a comprehensive temporal cascade in Drosophila medulla neuroblasts: Hth + SoxN + dmrt99B $->$ Opa $->$ Ey+Erm $\rightarrow$ Ey+Opa $->$ Slp+Scro $->$ D $->$ BarH1\&2->TII, Gcm (Figure 7B), and also illustrated several principles that might be conserved during the temporal patterning of neural stem cells.

\section{Initiation of the TTF cascade.}

Before this study, Hth was proposed to be the only TTF at play during the earliest temporal stage. Hth is expressed in the neuroepithelium and the youngest NBs. It is necessary for the generation of Bsh neurons, but is required neither for the NE to NB transition, nor for the further temporal cascade progression. Loss of Ey also does not affect the termination of Hth ${ }^{18,19}$. These data suggested missing links between $\mathrm{Hth}$ and the later TTF cascade. Here we identified several novel TTFs that linked the whole cascade together. Two novel TTFs that started their expression in the NE, SoxN and Dmrt99B, are also required for the first temporal fate (Bsh neurons), and Dmrt99B is required for the timely activation of Opa in the youngest NBs. Opa is then required to activate Ey and repress Hth (Figure 7A,B). Interestingly, the three TTFs inherited from NE maintain their expression for different durations in NBs, as Hth is repressed by Opa and Erm, SoxN is repressed by Ey, while Dmrt99B expression extends until the Slp stage (Figure7A, B). Whether this differential down-regulation is significant for temporal patterning is currently unknown. However, it is worth noting that the expression of mammalian orthologs of Dmrt99B,

Dmrt3 and Dmrta1, also starts in symmetrically dividing early cortical progenitors (NE), and 
decreases gradually in asymmetrical dividing cortical progenitors due to the direct suppression by FoxG1, the mammalian ortholog of SIp1/2 ${ }^{83,84}$. Given the essential role of Dmrt99B in initiating temporal patterning in medulla neuroblast, it will be interesting to investigate whether its mammalian orthologs play conserved roles in the temporal progression of cortical progenitors.

\section{A broad temporal stage can be divided into sub-temporal stages by combinations of TTFs.}

At certain temporal stages, not just one TTF, but combinations of TTFs determine the progeny fates. This is well-illustrated in the Ey stage. The first stripe of Opa is required to initiate Ey expression in NBs, and Erm (as shown by scRNA-seq and Erm::V5) is turned on at a similar time as Ey. Due to the lack of Erm antibody, we haven't examined how Erm expression is regulated, but judging from the expression pattern, it is possible that Erm activation might also require Opa. Erm then transiently represses Opa until itself is tuned off possibly due to the loss of Opa, forming a negative feedback loop. Negative feedback loops with time-delay can generate striped expression patterns. After Erm is turned off, Opa is turned back on. At the same time SIp has been gradually activated by Ey and Scro, and when it reaches a certain level, it will repress Opa and Ey to end the Ey stage. Thus, cross-regulations among TTFs divide the Ey stage into (at least) two (sub-)temporal stages determined by the co-expression of Ey and Erm, or Ey and Opa. We showed that different neural types are generated in these two sub-temporal stages, and the first set of neurons require both Ey and Erm, while the second set of neurons require both Ey and Opa

(Figure 7A,B). Interestingly to note, the mammalian ortholog of Erm, Fezf2, is also expressed in cortical progenitors and play important roles in cortical neuron specification ${ }^{34,35,85}$.

\section{Termination of the TTF cascade.}


Previously it was thought that TII stage NBs switch to gliogenesis and then exit the cell cycle, but whether Tll indeed plays a role in these processes has not been studied. Here our scRNA-Seq data suggested another final temporal stage marked by the expression of $\mathrm{Gcm}$ and Dap. Further we showed that BarH1 and BarH2 are required to activate both TII and Gcm, but with different kinetics. TII is activated first, and when $\mathrm{Gcm}$ is activated, Gcm represses Tll. We showed that $\mathrm{Gcm}$ but not TII is required for the NBs to switch to gliogenesis and exit the cell cycle (Figure 7 A,B). Gcm is well-known for its role in gliogenesis, but here we show that it is also required to activate Dap expression in neuroblasts to promote cell cycle exit and end the temporal progression. In vertebrate retina, scRNA-seq analysis of retinal progenitor cells identified NFI factors as required for both late-born cell fates including Müller glia and for exiting the cell cycle ${ }^{28}$. Since neural progenitors often switch to produce glia at the end of the lineage, it might be a general mechanism that factors required for the switch to gliogenesis are also required for the mitotic exit to end the temporal progression.

\section{Complex cross-regulations among TTFs form temporal gene networks.}

The model for the cross-regulations between medulla TTFs is that each TTF activates the next TTF and inhibits the previous TTF from the Ey stage to the end of the cascade, exhibiting a simple combination of feedforward activation and feedback repression. However, based on the experimental evidence we produced as well as inferred from the scRNA-seq data, the crossregulations among TTFs are more complex. One TTF is not necessarily repressed by the very next TTF, or activated by the exactly previous TTF. Hth is repressed by Opa and Erm. SoxN is repressed by Ey, while Dmrt99B is likely to be repressed by SIp or later TTFs. TIl is activated just before Gcm, 
however, TIl is not required for Gcm's activation. The complexity of their cross-regulation is a way to increase the number of combinations of TTFs in aging NBs, thereby increase the number of possible neuron fates determined along the temporal progression. However, the overall trend that early TTFs activate late TTFs, and late TTFs repress early TTFs remains valid.

\section{Genes functioning in the differentiation axis regulate the TTF cascade}

A novel mechanism for the regulation of temporal patterning is discovered in our study:

TFs differentially expressed and functioning along the differentiation axis also contribute to the regulation of the TTF cascade (Figure $6 \mathrm{P}, \mathrm{Q}$ ). There are two temporal axes in a NB lineage, one is the TTF cascade progression in NBs, and the other is the gradual differentiation of the progeny losing the self-renewal ability (NBs to GMCs to neurons). Whether the two axes interact with each other was not clear. In this study we investigated the role of two TFs, Lola and Nerfin-1, that are regulated and functioning along the differentiation axis, in the TTF cascade progression. Lola proteins belong to a BTB/POZ family of proteins which have been shown to be involved in chromatin remodeling and organization ${ }^{86}$. Certain isoforms of Lola are expressed in all NBs, e.g. Lola-F is activated one cell cycle earlier than Opa. We show that Lola proteins functions as a speed modulator of the temporal cascade progression. It represses the expression of Hth, facilitates the activation of Opa and the following TTFs to different extents, and guarantees a quick transition from the NE gene network to the NB gene network. Interestingly, the vertebrate ortholog of lola, Zbtb20, was also found to modulate the sequential generation of different neural types in cortical progenitors ${ }^{87}$. Loss of Zbtb20 causes the temporal transitions to be delayed further and further, 
very similar to the loss of lola phenotype in our system. Thus, it is possible that lola / Zbtb20 play conserved roles in temporal patterning of neural progenitors.

The expression of Nerfin-1 is also differentiation-axis-dependent. It is observable mostly in maturing neurons, and is required to prevent neurons from dedifferentiation ${ }^{81,82}$. However, this TF responsible for maintaining the differentiation status of neurons, is turned on in the final stage NBs, where it functions to promote gliogenesis and help terminate the temporal cascade on time. The fast exit of cell cycle at the final stage might be accomplished because self-renewal repressors that usually function in GMCs and neurons, such as Prospero ${ }^{18}$ and Nerfin-1, gather and cooperate in the oldest NBs. Since Gcm is also required to promote gliogenesis and cell cycle exit, Gcm and Nerfin-1 might regulate each other's expression or cooperate with each other in this process.

In summary, the entire life of a medulla neuroblast from the beginning to the end was revealed in this study. Our comprehensive study of the medulla neuroblast temporal cascade illustrated mechanisms that might be conserved in the temporal patterning of neural stem cells. The single cell RNA-sequencing data provide a plethora of information that allow further exploration of the mechanisms of temporal patterning.

\section{Methods}

\section{Fly lines and crosses.}

Construction of fly lines. To construct the stock for labeling of medulla neuroblasts for FACS sorting and scRNA-seq, SoxNGal4 (GMR41H10Gal4) ${ }^{88}$ was recombined with UAS-RedStinger 
(BDSC 8547) on Chromosome III, and then crossed with E(spl)myGFP on II ${ }^{89}$, to generate the E(spl)myGFP; SoxNGal4 UAS-RedStinger/TM6B stock. To generate SoxN mutant clones, SoxN ${ }^{N C 14}$ mutation (BDSC 9938) was recombined onto FRT40A chromosome, to generate the FRT40A SoxN $N^{N C 14}$ stock.

MARCM mutant clones with FRT40A mutants. To generate MARCM mutant clones of SoxN mutant, s/p mutant, erm mutant, or $\mathrm{gcm}$ gcm 2 double mutant, virgin females of $y w$ hsFLP UASCD8GFP; FRT40A tubGal80; tubGal4/TM6B were crossed with males of FRT40A SoxN $N^{N C 14} / C y O$, FRT4OA sIp S37A /SM6-TM6B (Gift from Andrew Tomlinson ${ }^{90}$ ), FRT4OA erm ${ }^{1} / C y O, G F P$ (gift from Cheng-Yu Lee ${ }^{66}$ ), or $D f(2 L) 200$ FRT4OA / Gla, Bc (which deletes both gcm and gcm2 ${ }^{78}$ ) respectively. The progeny were grown at $25^{\circ} \mathrm{C}$, heat shocked once at $37^{\circ} \mathrm{C}$ for $40 \mathrm{~min}$ at 1 st instar larval stage, and then grown at $25^{\circ} \mathrm{C}$ for three days before dissection of the wandering $3 r d$ instar larvae.

MARCM mutant clones with FRT82B mutants. To generate MARCM clones of $h$ th mutant or opa mutant, virgin females of ywhsFLP UASCD8GFP; ; tubGal4, FRT82B tubGal80/TM6B were crossed with FRT82B hth ${ }^{P 2} / T M 6 B$ flies (gifts from Richard Mann), FRT82B opa ${ }^{7}$ (gift from Deborah Hursh $\left.{ }^{63}\right)$, respectively. The progeny were grown at $25{ }^{\circ} \mathrm{C}$, heat shocked once at $37{ }^{\circ} \mathrm{C}$ for $1 \mathrm{hr}$ at 1 st instar larval stage, and then grown at $25^{\circ} \mathrm{C}$ for three days before dissection of the wandering $3 \mathrm{rd}$ instar larvae.

Negatively marked ey mutant clones. Females of $y w, h s-F l p^{1.22}$; FRT8OB, eyBAC, UbiGFP/TM6B,Tb; ey $y^{J 5.71}$ were crossed to males with genotype $h s-F l p^{1.22} ; ; F R T 80 B ; e y^{J 5.71} / \operatorname{In}(4) c i^{D}$ $\left(\right.$ Ref $\left.{ }^{18}\right)$. The progeny were grown at $25^{\circ} \mathrm{C}$, heat shocked once at $37^{\circ} \mathrm{C}$ for $1 \mathrm{hr}$ at 1 st instar larval stage, and then grown at $25^{\circ} \mathrm{C}$ for three days before dissection of the wandering $3 \mathrm{rd}$ instar larvae. 
Clones in larvae that lacked both GFP fluorescence and staining with an anti-Ey antibody were further analyzed.

RNAi clones. RNAi lines used in this study include: UAS-eyRNAi (BDSC 32486), UAS-dmrt99B-RNAi (BDSC 31982), UAS-opaRNAi (VDRC 101531), UAS-erm ${ }^{R N A i}$ (BDSC 50661), UAS-scro ${ }^{R N A i}$ lines (BDSC 29387, and BDSC 33890 showed the same phenotypes), UAS-D ${ }^{\text {RNAi }}$ (VDRC 107194), UAS-BarH1 ${ }^{\text {RNAi }}$ (VDRC 104681), UAS-BarH2 ${ }^{R N A i}$ (VDRC 11570), UAS-tIl-miRNA (gift from Tzumin Lee ${ }^{91}$ ), UAS$\mathrm{gcm}^{R N A i}$ (VDRC 110539, VDRC 2961 showed the same phenotypes), UAS-lola ${ }^{R N A i}$ lines (BDSC 35721, BDSC 26714, VDRC 101925, all showed similar phenotypes), UAS-nerfin-1 ${ }^{R N A i}$ (VDRC 101631), UAS-oaz $^{\text {RNAi }}$ (VDRC 39214, VDRC 107061), UAS-hbn ${ }^{\text {RNAi }}$ (VDRC 103979), UAS-sba ${ }^{\text {RNAi }}$ (vdrc 101314), and UAS-scrt ${ }^{R N A i}$ (VDRC 105201). To generate RNAi clones, virgin females of yw hs FLP; act>y+>Gal4 UAS GFP / CyO; UASDCR2/TM6B were crossed with males of each of the RNAi lines. The progeny were grown at $25^{\circ} \mathrm{C}$, heat shocked once at $37^{\circ} \mathrm{C}$ for $8 \mathrm{~min}$ at $1^{\text {st }}$ instar larval stage, and transferred to $29^{\circ} \mathrm{C}$ for three days before dissection of the wandering $3^{\text {rd }}$ instar larvae.

Region-specific RNAi. Alternatively, region-specific Gal4s combined with UAS-DCR2 were used to drive RNAi. Virgin females of UAS-Dcr2;Dpn-LacZ/CyO; VsxGal4/TM6B ${ }^{65}$ were crossed with males of UAS-opaRNAi (VDRC 101531). Virgin females of UASDCR2; optixGal4/CyO ${ }^{65}$ were crossed with UAS-Iola ${ }^{R N A i}$ lines (BDSC 26714, BDSC 35721). The progeny were grown at $25^{\circ} \mathrm{C}$ until $1^{\text {st }}$ instar larval stage and transferred to $29^{\circ} \mathrm{C}$ for three days before dissection of the wandering $3^{\text {rd }}$ instar larvae.

GFP-BAC and other reporter lines. Additional lines used in this study include Dmrt99B::GFP (BDSC 81280), Erm::V5 (gift from Cheng-Yu Lee ${ }^{69}$ ), a ${ }^{\text {rk568 }}-l a c Z{ }^{92}$, B-H2::GFP (BDSC 67734), Gcm::GFP

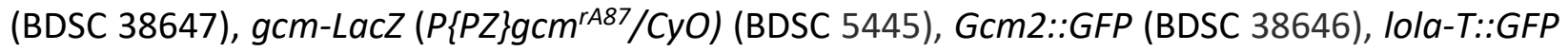


(flybase name: lola.GR-GFP) (BDSC: 38661), lola-K::GFP (flybase name: lola.I-GFP) (BDSC: 38662), and Nerfin-1::GFP(BDSC 67385).

\section{Dissociation and FACS sorting of medulla neuroblasts}

For each scRNA-seq experiment, 120 third instar larvae of the genotype $E(s p l) m y G F P$; SoxNGal4 UAS-RedStinger /TM6B were washed with PBS twice, and with 70\% ethanal for 1 min, and with PBS once again. Each of the brains was dissected on ice in complete Schneider's culture medium (Schneider's Insect medium, supplemented with 10\% fetal bovine serum, 2\% Pen/Strep and $0.02 \mathrm{mg} / \mathrm{mL}$ insulin), and was directly transferred into a glass dish on ice containing DPBS. The dissection was completed within one hour, and then the supernatant (mainly DPBS) was replaced by $1 \mathrm{~mL}$ TrypLE with $1 \mathrm{mg} / \mathrm{mL}$ collagenase I and $1 \mathrm{mg} / \mathrm{mL}$ papain. The brains were incubated for $10 \mathrm{~min}$ at $30^{\circ} \mathrm{C}$, with gentle shaking at $55 \mathrm{rpm}$. After removal of the dissociation solution, the brains were carefully washed with complete Schneider's culture medium once and with DPBS twice. The brains were disrupted in $1.4 \mathrm{ml}$ of DPBS with $0.04 \%$ BSA by manual pipetting using a P1000, and then $0.4 \mathrm{ml}$ of DPBS with $0.04 \%$ BSA was added to make a total volume of 1.8 $\mathrm{mL}$. The cell suspension was filtered through the cell strainer cap into a $5 \mathrm{~mL}$ BDFalcon FACS tube. FACS sorting was done immediately after on BD FACS ARIA II with gentle settings ( $85 \mu \mathrm{m}$ nozzle and low pressure of 20 psi). Trypan blue was added before sorting. Among the singlet live cells, GFP and RFP double positive cells were selected, and sorted into DPBS with $0.04 \%$ BSA.

For immunohistochemistry of unsorted cells or sorted cells after concentration, the cell suspension was placed onto a coated (poly-D- lysine) dish for 30 minutes. After fixation with $4 \%$ formaldehyde for 10 minutes, the coverslip was washed 4 times with PBS. The primary antibodies were incubated for $2 \mathrm{hr}$, and was washed 3 times with PBST. Secondary antibodies were 
incubated for 30 min, and was washed 3 times with PBST. The cells were mounted in mounting medium and imaged on Zeiss confocal.

\section{Construction and sequencing of 10x V3 Single Cell libraries}

Single-cell 3' cDNA libraries were prepared and sequenced at the DNA Services laboratory of the Roy J. Carver Biotechnology Center at the University of Illinois at Urbana-Champaign. FACS sorted cells were immediately concentrated by centrifugation at $500 \mathrm{~g}$ for 5 minutes, then an additional $800 \mathrm{~g}$ for 5 minutes to a $40 \mathrm{ul}$ volume. This entire volume was used as input for the $10 \mathrm{x}$ library. The single cell suspension was converted into an individually barcoded cDNA library with the Chromium Next GEM Single-Cell 3' single-index kit version 3 from 10X Genomics (Pleasanton, CA) following the manufacturer's protocols. The target capture was $10 \mathrm{k}$ cells.

Following ds-cDNA synthesis, sequencing library compatible with the Illumina chemistry was constructed. The final library was quantitated on Qubit and the average size determined on the AATI Fragment Analyzer (Advanced Analytics, Ames, IA). The final library was diluted to 5nM concentration and further quantitated by qPCR on a Bio-Rad CFX Connect Real-Time System (BioRad Laboratories, Inc. CA).

The final library was sequenced on one lane of an Illumina NovaSeq 6000 S1 flowcell (exp1) or a half lane of an Illumina NovaSeq 6000 S4 flowcell, as paired-reads with 28 cycles for read 1, 8 cycles for the index read, and 150 cycles for read 2. Basecalling and demultiplexing of raw data was done with the mkfastq command of the software Cell Ranger 3.1.0 (10x Genomics). Libraries were sequenced to a depth of 2,019,439,522 total reads (1st exp.) and 
2,760,057,420 total reads ( 2 nd exp.), corresponding to 6548 cells with a 1821 median UMI counts per cell (1st exp), and 5343 cells with 7508 median UMI counts per cell (2nd exp).

\section{scRNA-seq analysis}

The sequencing reads were aligned to Ensembl's BDGP6.22 using Cell Ranger (version3.0.1 for $1^{\text {st }}$ experiment, and version 3.1.0 for $2^{\text {nd }}$ experiment) from $10 x$ Genomics, and gene expression levels were counted using Cellranger "Count". In both versions of Cell Ranger, "EmptyDrops" method ${ }^{93}$ was used to call cells.

All subsequent analyses were performed in R (version 4.0.3) ${ }^{94}$ Quality control on the count data was performed using the package Seurat (version 3.2 .3$)^{46}$. To limit the analysis to neuroblasts, only cells expressing Dpn were analyzed. Cells were also excluded if $10 \%$ or more of their reads came from mitochondrial genes. This left 777 cells from the first experiment and 2302 cells from the second experiment.

Data from both experiments were combined into a single analysis. Batch correction was performed using the standard integration workflow in Seurat. Specifically, each dataset was first separately normalized and the top 2000 most variable features were identified. These features were then used to find integration anchors, after which the data were combined using the IntegrateData function. Finally, five outlier cells with large numbers of counts were removed. The remaining 3074 cells were scaled and centered for downstream analysis.

UMAP coordinates were calculated with the RunUMAP function using the top 10 principal components of the 2000 most variable features. Clustering was performed using the FindNeighbors and FindClusters functions with a resolution of 0.9. Cell cycle scoring was performed using the CellCycleScoring function; see Supplementary Table 1 for lists of the S genes 
and G2/M genes used. Gene expression levels were visualized with the FeaturePlot function. Differentially expressed genes were identified using the FindAllMarkers function with an FDR cutoff of 0.05 .

Developmental trajectories were inferred using the Monocle3 (version 0.2.3.0) ${ }^{48-50}$ excluding the outlying clusters $8,11,12$, and 14 . Raw counts data were imported from the integrated Seurat object into a Monocle3 cell dataset object. Preprocessing was performed with the preprocess_cds function and batch correction was performed using the Batchelor algorithm 95 as implemented in the align_cds function. PCA and UMAP embeddings stored in the Monocle3 object were replaced with the corresponding values calculated by Seurat and stored in the Seurat object. Cells were then clustered in Monocle3 using the cluster_cells function and the principal graph was learned using the learn_graph function with the minimal branch length option set to 5. The root node was set to be the vertex closest to cells with the highest median Hth expression. Finally, pseudotimes were calculated using the order_cells function and the trajectory was visualized using the plot_cells function. Genes with temporally patterned expression gradients were identified as those whose expression levels showed significant Spearman correlation with the inferred pseudotime at an FDR cutoff of 0.05 . The top 200 genes with decreasing or increasing gradients, respectively, were analyzed for enriched Go terms for Biological Processes "GOTERM_BP_DIRECT" using the "Functional Annotation Chart" at the DAVID Bioinformatics Resources 6.8 website (https://david.ncifcrf.gov/home.jsp).

\section{Antibodies and Immunostaining}


These antibodies are generous gifts from the fly community: Rabbit anti-SoxN (1:100) from Steven Russell 55; Rabbit anti-Hth (1:500) from Richard Mann; Guinea-pig anti-Run, Rabbit anti-Bsh, Rabbit anti-SIp1, Guinea-pig anti-SIp2, Rabbit anti-D, Guinea-pig anti-TIl and Rabbit antiSox102F (all used at 1:500) from Claude Desplan; Rabbit anti-D (1:1000) from John R. Nambu ${ }^{96}$; Rat anti-Dfr (1:200) from Makoto Sato ${ }^{57}$, Guinea-pig anti-Kn (1:500) from Adrian Moore ${ }^{97}$, Guinea-pig anti-Dpn (1:500) from Chris Doe; Rabbit anti Opa (1:100) from J. Peter Gergen ${ }^{61}$; Rat anti-BarH1 (1: 200) from Tiffany Cook ${ }^{98}$.

Commercially available antibodies include: sheep anti-GFP (1:500, AbD Serotec, 47451051), Goat anti-beta-gal (1:1000, Abcam, ab12081), rabbit anti-RFP (1:1000, Abcam, ab62341), Mouse anti V5-Tag:DyLight ${ }^{\circledR 5} 50$ (1:200, Bio-Rad, MCA1360D550GA), Rat anti-Deadpan [11D1BC7] (1:200, Abcam, ab195173). These antibodies are provided by the Developmental Studies Hybridoma Bank (DSHB): mouse anti-eyeless (1:10), mouse anti-Pros (MR1A 1:10), mouse antiRepo (8D12 anti-Repo 1:50), and mouse anti-Dac (mAbdac2-3, 1:20) , mouse anti-Dap (NP1, 1:5), mouse anti-Lola -F (7F1-1D5, 1:20).

Secondary antibodies are from Jackson or Invitrogen. Immunostaining was done as described ${ }^{18}$ with a few modifications: $3^{\text {rd }}$ instar Larval brains were dissected in 1XPBS, and fixed in $4 \%$ Formaldehyde for 30 minutes on ice. Brains were washed and then incubated in primary antibody solution overnight at $4^{\circ} \mathrm{C}$, washed three times and incubated in secondary antibody solution overnight at $4^{\circ} \mathrm{C}$, washed three times and mounted in Slowfade. Images are acquired using a Zeiss Confocal Microscope. Figures are assembled using Photoshop and Illustrator.

\section{Quantifications and Statistical Analysis}


For each experiment, the numbers of animals or clones analyzed are included in each figure's legends. Sample sizes are estimated based on previous experience. Clonal experiments have internal controls: we compared gene expression in and outside of the clones in the same sample, and only draw conclusions when consistent results are obtained. For quantification of mng number in eyGal4 and eyGal4>Nerfin-1 RNAi brains, mng marked by Repo were counted on representative focal planes in 5 brains per genotype, and the $p$ value is calculated using student t-test.

\section{Data Availability}

The raw and processed scRNA-seq data have been deposited to GEO, and the accession number is GSE168553.

\section{References}

1. Lin, S. \& Lee, T. Generating neuronal diversity in the Drosophila central nervous system. Developmental Dynamics (2012) doi:10.1002/dvdy.22739.

2. Allan, D. W. \& Thor, S. Transcriptional selectors, masters, and combinatorial codes: Regulatory principles of neural subtype specification. Wiley Interdisciplinary Reviews: Developmental Biology (2015) doi:10.1002/wdev.191.

3. Holguera, I. \& Desplan, C. Neuronal specification in space and time. Science (2018) doi:10.1126/science.aas9435.

4. Sagner, A. \& Briscoe, J. Establishing neuronal diversity in the spinal cord: A time and a place. Development (Cambridge) (2019) doi:10.1242/dev.182154.

5. Doe, C. Q. Temporal Patterning in the Drosophila CNS . Annu. Rev. Cell Dev. Biol. (2017) doi:10.1146/annurev-cellbio-111315-125210.

6. Miyares, R. L. \& Lee, T. Temporal control of Drosophila central nervous system development. Current Opinion in Neurobiology (2019) doi:10.1016/j.conb.2018.10.016.

7. Isshiki, T., Pearson, B., Holbrook, S. \& Doe, C. Q. Drosophila neuroblasts sequentially express transcription factors which specify the temporal identity of their neuronal progeny. Cell (2001) doi:10.1016/S0092-8674(01)00465-2.

8. Kambadur, R. et al. Regulation of POU genes by castor and hunchback establishes layered 
compartments in the Drosophila CNS. Genes Dev. (1998) doi:10.1101/gad.12.2.246.

9. Brody, T. \& Odenwald, W. F. Programmed transformations in neuroblast gene expression during Drosophila CNS lineage development. Dev. Biol. (2000)

doi:10.1006/dbio.2000.9829.

10. Grosskortenhaus, R., Pearson, B. J., Marusich, A. \& Doe, C. Q. Regulation of temporal identity transitions in drosophila neuroblasts. Dev. Cell (2005)

doi:10.1016/j.devcel.2004.11.019.

11. Grosskortenhaus, R., Robinson, K. J. \& Doe, C. Q. Pdm and Castor specify late-born motor neuron identity in the NB7-1 lineage. Genes Dev. (2006) doi:10.1101/gad.1445306.

12. Baumgardt, M., Karlsson, D., Terriente, J., Díaz-Benjumea, F. J. \& Thor, S. Neuronal Subtype Specification within a Lineage by Opposing Temporal Feed-Forward Loops. Cell (2009) doi:10.1016/j.cell.2009.10.032.

13. Maurange, C., Cheng, L. \& Gould, A. P. Temporal Transcription Factors and Their Targets Schedule the End of Neural Proliferation in Drosophila. Cell (2008) doi:10.1016/j.cell.2008.03.034.

14. Liu, Z. et al. Opposing intrinsic temporal gradients guide neural stem cell production of varied neuronal fates. Science (80-. ). (2015) doi:10.1126/science.aad1886.

15. Ren, Q. et al. Stem Cell-Intrinsic, Seven-up-Triggered Temporal Factor Gradients Diversify Intermediate Neural Progenitors. Curr. Biol. (2017) doi:10.1016/j.cub.2017.03.047.

16. Syed, M. H., Mark, B. \& Doe, C. Q. Steroid hormone induction of temporal gene expression in drosophila brain neuroblasts generates neuronal and glial diversity. Elife (2017) doi:10.7554/eLife.26287.

17. Pahl, M. C., Doyle, S. E. \& Siegrist, S. E. E93 Integrates Neuroblast Intrinsic State with Developmental Time to Terminate MB Neurogenesis via Autophagy. Curr. Biol. (2019) doi:10.1016/j.cub.2019.01.039.

18. Li, X. et al. Temporal patterning of Drosophila medulla neuroblasts controls neural fates. Nature 498, 456-462 (2013).

19. Suzuki, T., Kaido, M., Takayama, R. \& Sato, M. A temporal mechanism that produces neuronal diversity in the Drosophila visual center. Dev. Biol. (2013) doi:10.1016/j.ydbio.2013.05.002.

20. Bertet, C. et al. Temporal patterning of neuroblasts controls notch-mediated cell survival through regulation of hid or reaper. Cell (2014) doi:10.1016/j.cell.2014.07.045.

21. Bayraktar, O. A. \& Doe, C. Q. Combinatorial temporal patterning in progenitors expands neural diversity. Nature (2013) doi:10.1038/nature12266.

22. Eroglu, E. et al. SWI/SNF complex prevents lineage reversion and induces temporal patterning in neural stem cells. Cell (2014) doi:10.1016/j.cell.2014.01.053.

23. Abdusselamoglu, M. D., Eroglu, E., Burkard, T. R. \& Knoblich, J. A. The transcription factor odd-paired regulates temporal identity in transit- amplifying neural progenitors via an incoherent feed-forward loop. Elife (2019) doi:10.7554/eLife.46566.

24. Maurange, C. Temporal patterning in neural progenitors: From Drosophila development to childhood cancers. DMM Disease Models and Mechanisms (2020) doi:10.1242/dmm.044883.

25. Oberst, P., Agirman, G. \& Jabaudon, D. Principles of progenitor temporal patterning in the developing invertebrate and vertebrate nervous system. Current Opinion in 
Neurobiology (2019) doi:10.1016/j.conb.2019.03.004.

26. Elliott, J., Jolicoeur, C., Ramamurthy, V. \& Cayouette, M. Ikaros Confers Early Temporal Competence to Mouse Retinal Progenitor Cells. Neuron (2008) doi:10.1016/j.neuron.2008.08.008.

27. Mattar, P., Ericson, J., Blackshaw, S. \& Cayouette, M. A conserved regulatory logic controls temporal identity in mouse neural progenitors. Neuron (2015) doi:10.1016/j.neuron.2014.12.052.

28. Clark, B. S. et al. Single-Cell RNA-Seq Analysis of Retinal Development Identifies NFI Factors as Regulating Mitotic Exit and Late-Born Cell Specification. Neuron (2019) doi:10.1016/j.neuron.2019.04.010.

29. Liu, S. et al. Foxn4 is a temporal identity factor conferring mid/late-early retinal competence and involved in retinal synaptogenesis. Proc. Natl. Acad. Sci. U. S. A. (2020) doi:10.1073/pnas.1918628117.

30. Hanashima, C., Li, S. C., Shen, L., Lai, E. \& Fishell, G. Foxg1 Suppresses Early Cortical Cell Fate. Science (80-. ). (2004) doi:10.1126/science.1090674.

31. Alsiö, J. M., Tarchini, B., Cayouette, M. \& Livesey, F. J. Ikaros promotes early-born neuronal fates in the cerebral cortex. Proc. Natl. Acad. Sci. U. S. A. (2013) doi:10.1073/pnas.1215707110.

32. Naka, H., Nakamura, S., Shimazaki, T. \& Okano, H. Requirement for COUP-TFI and II in the temporal specification of neural stem cells in CNS development. Nat. Neurosci. (2008) doi:10.1038/nn.2168.

33. Pearson, C. A. et al. Foxp1 Regulates Neural Stem Cell Self-Renewal and Bias Toward Deep Layer Cortical Fates. Cell Rep. (2020) doi:10.1016/j.celrep.2020.01.034.

34. Chen, B., Schaevitz, L. R. \& McConnell, S. K. Fezl regulates the differentiation and axon targeting of layer 5 subcortical projection neurons in cerebral cortex. Proc. Natl. Acad. Sci. U. S. A. (2005) doi:10.1073/pnas.0508732102.

35. Chen, B. et al. The Fezf2-Ctip2 genetic pathway regulates the fate choice of subcortical projection neurons in the developing cerebral cortex. Proc. Natl. Acad. Sci. U. S. A. (2008) doi:10.1073/pnas.0804918105.

36. Telley, L. et al. Temporal patterning of apical progenitors and their daughter neurons in the developing neocortex. Science (80-. ). (2019) doi:10.1126/science.aav2522.

37. Yasugi, T., Umetsu, D., Murakami, S., Sato, M. \& Tabata, T. Drosophila optic lobe neuroblasts triggered by a wave of proneural gene expression that is negatively regulated by JAK/STAT. Development (2008) doi:10.1242/dev.019117.

38. Yasugi, T., Sugie, A., Umetsu, D. \& Tabata, T. Coordinated sequential action of EGFR and Notch signaling pathways regulates proneural wave progression in the Drosophila optic lobe. Development (2010) doi:10.1242/dev.048058.

39. Egger, B., Gold, K. S. \& Brand, A. H. Notch regulates the switch from symmetric to asymmetric neural stem cell division in the Drosophila optic lobe. Development (2010) doi:10.1242/dev.051250.

40. Naidu, V. G. et al. Temporal progression of Drosophila medulla neuroblasts generates the transcription factor combination to control T1 neuron morphogenesis. Dev. Biol. (2020) doi:10.1016/j.ydbio.2020.05.005.

41. Soustelle, L. \& Giangrande, A. Novel gcm-dependent lineages in the postembryonic 
nervous system of Drosophila melanogaster. Dev. Dyn. (2007) doi:10.1002/dvdy.21232.

42. Jeong, H. \& Tiwari, V. K. Exploring the complexity of cortical development using singlecell transcriptomics. Frontiers in Neuroscience (2018) doi:10.3389/fnins.2018.00031.

43. Bates, A. S., Janssens, J., Jefferis, G. S. \& Aerts, S. Neuronal cell types in the fly: single-cell anatomy meets single-cell genomics. Current Opinion in Neurobiology (2019) doi:10.1016/j.conb.2018.12.012.

44. Konstantinides, N., Degabriel, S. \& Desplan, C. Neuro-evo-devo in the single cell sequencing era. Current Opinion in Systems Biology (2018) doi:10.1016/j.coisb.2018.08.001.

45. Lopes, A., Magrinelli, E. \& Telley, L. Emerging roles of single-cell multi-omics in studying developmental temporal patterning. International Journal of Molecular Sciences (2020) doi:10.3390/ijms21207491.

46. Butler, A., Hoffman, P., Smibert, P., Papalexi, E. \& Satija, R. Integrating single-cell transcriptomic data across different conditions, technologies, and species. Nat. Biotechnol. (2018) doi:10.1038/nbt.4096.

47. Mclnnes, L., Healy, J. \& Melville, J. UMAP: Uniform manifold approximation and projection for dimension reduction. arXiv (2018).

48. Trapnell, C. et al. The dynamics and regulators of cell fate decisions are revealed by pseudotemporal ordering of single cells. Nat. Biotechnol. (2014) doi:10.1038/nbt.2859.

49. Qiu, X. et al. Reversed graph embedding resolves complex single-cell trajectories. Nat. Methods (2017) doi:10.1038/nmeth.4402.

50. Cao, J. et al. The single-cell transcriptional landscape of mammalian organogenesis. Nature (2019) doi:10.1038/s41586-019-0969-x.

51. Hens, K. et al. Automated protein-DNA interaction screening of Drosophila regulatory elements. Nat. Methods (2011) doi:10.1038/nmeth.1763.

52. Buescher, M., Hing, F. S. \& Chia, W. Formation of neuroblasts in the embryonic central nervous system of Drosophila melanogaster is controlled by SoxNeuro. Development (2002).

53. Bahrampour, S., Gunnar, E., Jonsson, C., Ekman, H. \& Thor, S. Neural Lineage Progression Controlled by a Temporal Proliferation Program. Dev. Cell (2017)

doi:10.1016/j.devcel.2017.10.004.

54. Arefin, B., Parvin, F., Bahrampour, S., Stadler, C. B. \& Thor, S. Drosophila Neuroblast Selection Is Gated by Notch, Snail, SoxB, and EMT Gene Interplay. Cell Rep. (2019) doi:10.1016/j.celrep.2019.11.038.

55. Ferrero, E., Fischer, B. \& Russell, S. SoxNeuro orchestrates central nervous system specification and differentiation in Drosophila and is only partially redundant with Dichaete. Genome Biol. (2014) doi:10.1186/gb-2014-15-5-r74.

56. Lee, T. \& Luo, L. Mosaic analysis with a repressible cell marker (MARCM) for Drosophila neural development. Trends in Neurosciences (2001) doi:10.1016/S0166-2236(00)017914.

57. Hasegawa, E. et al. Concentric zones, cell migration and neuronal circuits in the Drosophila visual center. Development (2011) doi:10.1242/dev.058370.

58. Ito, K., Awano, W., Suzuki, K., Hiromi, Y. \& Yamamoto, D. The Drosophila mushroom body is a quadruple structure of clonal units each of which contains a virtually identical set of 
neurones and glial cells. Development (1997).

59. Henry, G. L., Davis, F. P., Picard, S. \& Eddy, S. R. Cell type-specific genomics of Drosophila neurons. Nucleic Acids Res. (2012) doi:10.1093/nar/gks671.

60. Fontana, J. R. \& Crews, S. T. Transcriptome analysis of Drosophila CNS midline cells reveals diverse peptidergic properties and a role for castor in neuronal differentiation. Dev. Biol. (2012) doi:10.1016/j.ydbio.2012.09.010.

61. Koromila, T. et al. Odd-paired is a pioneer-like factor that coordinates with zelda to control gene expression in embryos. Elife (2020) doi:10.7554/eLife.59610.

62. Soluri, I. V., Zumerling, L. M., Parra, O. A. P., Clark, E. G. \& Blythe, S. A. Zygotic pioneer factor activity of odd-paired/zic is necessary for late function of the drosophila segmentation network. Elife (2020) doi:10.7554/eLife.53916.

63. Lee, H., Stultz, B. G. \& Hursh, D. A. The Zic family member, odd-paired, regulates the Drosophila BMP, decapentaplegic, during adult head development. Development (2007) doi:10.1242/dev.02807.

64. Hursh, D. A. \& Stultz, B. G. Odd-Paired: The drosophila Zic gene. in Advances in Experimental Medicine and Biology (2018). doi:10.1007/978-981-10-7311-3_3.

65. Erclik, T. et al. Integration of temporal and spatial patterning generates neural diversity. Nature (2017) doi:10.1038/nature20794.

66. Weng, M., Golden, K. L. \& Lee, C. Y. dFezf/Earmuff Maintains the Restricted Developmental Potential of Intermediate Neural Progenitors in Drosophila. Dev. Cell (2010) doi:10.1016/j.devcel.2009.12.007.

67. Janssens, D. H. et al. Earmuff restricts progenitor cell potential by attenuating the competence to respond to self-renewal factors. Dev. (2014) doi:10.1242/dev.106534.

68. Koe, C. T. et al. The Brm-HDAC3-Erm repressor complex suppresses dedifferentiation in Drosophila type II neuroblast lineages. Elife (2014) doi:10.7554/eLife.01906.

69. Rives- Quinto, N. et al. Sequential activation of transcriptional repressors promotes progenitor commitment by silencing stem cell identity genes. Elife (2020) doi:10.7554/elife.56187.

70. Courgeon, M. \& Desplan, C. Coordination between stochastic and deterministic specification in the Drosophila visual system. Science (80-. ). (2019) doi:10.1126/science.aay6727.

71. Yoo, S. et al. Knock-in mutations of scarecrow, a Drosophila homolog of mammalian Nkx2.1, reveal a novel function required for development of the optic lobe in Drosophila melanogaster. Dev. Biol. (2020) doi:10.1016/j.ydbio.2020.02.008.

72. Zaffran, S., Das, G. \& Frasch, M. The NK-2 homeobox gene scarecrow (scro) is expressed in pharynx, ventral nerve cord and brain of Drosophila embryos. Mech. Dev. (2000) doi:10.1016/S0925-4773(00)00298-7.

73. Higashijima, S. I. et al. Dual Bar homeo box genes of Drosophila required in two photoreceptor cells, R1 and R6, and primary pigment cells for normal eye development. Genes Dev. (1992) doi:10.1101/gad.6.1.50.

74. Hayashi, T., Kojima, T. \& Saigo, K. Specification of primary pigment cell and outer photoreceptor fates by BarH1 homeobox gene in the developing Drosophila eye. Dev. Biol. (1998) doi:10.1006/dbio.1998.8959.

75. Hosoya, T., Takizawa, K., Nitta, K. \& Hotta, Y. Glial cells missing: A binary switch between 
neuronal and glial determination in drosophila. Cell (1995) doi:10.1016/00928674(95)90281-3.

76. Jones, B. W., Fetter, R. D., Tear, G. \& Goodman, C. S. glial cells missing: a genetic switch that controls glial versus neuronal fate. Cell (1995) doi:10.1016/0092-8674(95)90280-5.

77. Vincent, S., Vonesch, J. L. \& Giangrande, A. Glide directs glial fate commitment and cell fate switch between neurones and glia. Development (1996).

78. Chotard, C., Leung, W. \& Salecker, I. glial cells missing and gcm2 cell autonomously regulate both glial and neuronal development in the visual system of Drosophila. Neuron (2005) doi:10.1016/j.neuron.2005.09.019.

79. Dinges, N., Morin, V., Kreim, N., Southall, T. D. \& Roignant, J. Y. Comprehensive Characterization of the Complex lola Locus Reveals a Novel Role in the Octopaminergic Pathway via Tyramine Beta-Hydroxylase Regulation. Cell Rep. (2017) doi:10.1016/j.celrep.2017.11.015.

80. Southall, T. D., Davidson, C. M., Miller, C., Carr, A. \& Brand, A. H. Dedifferentiation of neurons precedes tumor formation in lola mutants. Dev. Cell (2014) doi:10.1016/j.devcel.2014.01.030.

81. Froldi, F. et al. The transcription factor Nerfin-1 prevents reversion of neurons into neural stem cells. Genes Dev. (2015) doi:10.1101/gad.250282.114.

82. Xu, J. et al. Prevention of medulla neuron dedifferentiation by nerfin-1 requires inhibition of notch activity. Dev. (2017) doi:10.1242/dev.141341.

83. Kumamoto, T. et al. Foxg1 coordinates the switch from nonradially to radially migrating glutamatergic subtypes in the neocortex through spatiotemporal repression. Cell Rep. (2013) doi:10.1016/j.celrep.2013.02.023.

84. Okamoto, M. et al. Cell-cycle-independent transitions in temporal identity of mammalian neural progenitor cells. Nat. Commun. (2016) doi:10.1038/ncomms11349.

85. Muralidharan, B. et al. LHX2 interacts with the NuRD complex and regulates cortical neuron subtype determinants Fezf2 and Sox11. J. Neurosci. (2017) doi:10.1523/JNEUROSCI.2836-16.2016.

86. Chaharbakhshi, E. \& Jemc, J. C. Broad-complex, tramtrack, and bric-à-brac (BTB) proteins: Critical regulators of development. Genesis (2016) doi:10.1002/dvg.22964.

87. Tonchev, A. B., Tuoc, T. C., Rosenthal, E. H., Studer, M. \& Stoykova, A. Zbtb20 modulates the sequential generation of neuronal layers in developing cortex. Mol. Brain (2016) doi:10.1186/s13041-016-0242-2.

88. Pfeiffer, B. D. et al. Tools for neuroanatomy and neurogenetics in Drosophila. Proc. Natl. Acad. Sci. U. S. A. (2008) doi:10.1073/pnas.0803697105.

89. Almeida, M. S. \& Bray, S. J. Regulation of post-embryonic neuroblasts by Drosophila Grainyhead. Mech. Dev. (2005) doi:10.1016/j.mod.2005.08.004.

90. Sato, A. \& Tomlinson, A. Dorsal-ventral midline signaling in the developing Drosophila eye. Development (2007) doi:10.1242/dev.02786.

91. Lin, S., Huang, Y. \& Lee, T. Nuclear receptor unfulfilled regulates axonal guidance and cell identity of Drosophila mushroom body neurons. PLoS One (2009) doi:10.1371/journal.pone.0008392.

92. Cohen, B., McGuffin, M. E., Pfeifle, C., Segal, D. \& Cohen, S. M. apterous, a gene required for imaginal disc development in Drosophila encodes a member of the LIM family of 
developmental regulatory proteins. Genes Dev. (1992) doi:10.1101/gad.6.5.715.

93. Lun, A. T. L. et al. EmptyDrops: Distinguishing cells from empty droplets in droplet-based single-cell RNA sequencing data. Genome Biol. (2019) doi:10.1186/s13059-019-1662-y.

94. R Core Team (2020). R: A language and environment for statistical computing. $R$ : $A$ language and environment for statistical computing. $R$ Foundation for Statistical Computing, Vienna, Austria (2020).

95. Haghverdi, L., Lun, A. T. L., Morgan, M. D. \& Marioni, J. C. Batch effects in single-cell RNAsequencing data are corrected by matching mutual nearest neighbors. Nat. Biotechnol. (2018) doi:10.1038/nbt.4091.

96. Ma, Y. et al. Gene regulatory functions of Drosophila Fish-hook, a high mobility group domain Sox protein. Mech. Dev. (1998) doi:10.1016/S0925-4773(98)00050-1.

97. Jinushi-Nakao, S. et al. Knot/Collier and Cut Control Different Aspects of Dendrite Cytoskeleton and Synergize to Define Final Arbor Shape. Neuron (2007) doi:10.1016/j.neuron.2007.10.031.

98. Charlton-Perkins, M. et al. Prospero and Pax2 combinatorially control neural cell fate decisions by modulating Ras- and Notch-dependent signaling. Neural Dev. (2011) doi:10.1186/1749-8104-6-20.

\section{Acknowledgments}

We thank the Flow Cytometry Facility and the DNA Services Laboratory, and the High Performance Computing in Biology Group of the Roy J. Carver Biotechnology Center at the University of Illinois at Urbana-Champaign for FACS sorting, and for construction and sequencing of the 10x V3 Single Cell libraries, and initial analysis of the sequencing data, respectively, and for providing the corresponding method part. We thank the fly community, especially Claude Desplan, Steven Russell, Cheng-Yu Lee, Tzumin Lee, Deborah Hursh, J. Peter Gergen, Tiffany Cook, Adrian Moore, Chris Doe; Richard Mann, Andrew Tomlinson, John R. Nambu and Makoto Sato, for generous gifts of antibodies and fly stocks. We thank the Bloomington Drosophila Stock Center, the Vienna Drosophila RNAi Center, the Developmental Studies Hybridoma Bank, and TriP at Harvard Medical School (NIH/NIGMS R01-GM084947) for fly stocks and reagents. We 
would like to thank the NSF-Simons Center for Quantitative Biology at Northwestern University for supporting this project as a Pilot grant, and also the National Eye Institute for grant support (Grant 1 R01 EY026965-01A1).

\section{Author contributions.}

X.L. and H.Z. designed the project and experiments, S.D.Z. analyzed sc-RNA-seq data, H.Z. and X.L. performed experiments and analyzed data, A.R. and Y.Z. participated in some experiments and data discussion. The manuscript is written by H.Z. , X.L. and S.D.Z., with all authors commented. Competing interests.

The authors declare no competing or financial interests.

Materials \& Correspondence. Publicly available fly lines (those from BDSC or VDRC) should be requested directly from the corresponding stock centers: https://bdsc.indiana.edu/ or https://stockcenter.vdrc.at/control/main. Fly lines generated in this study can be requested without restriction. Correspondence and requests for materials should be addressed to Xin Li (lixin@illinois.edu). 
Figure 1

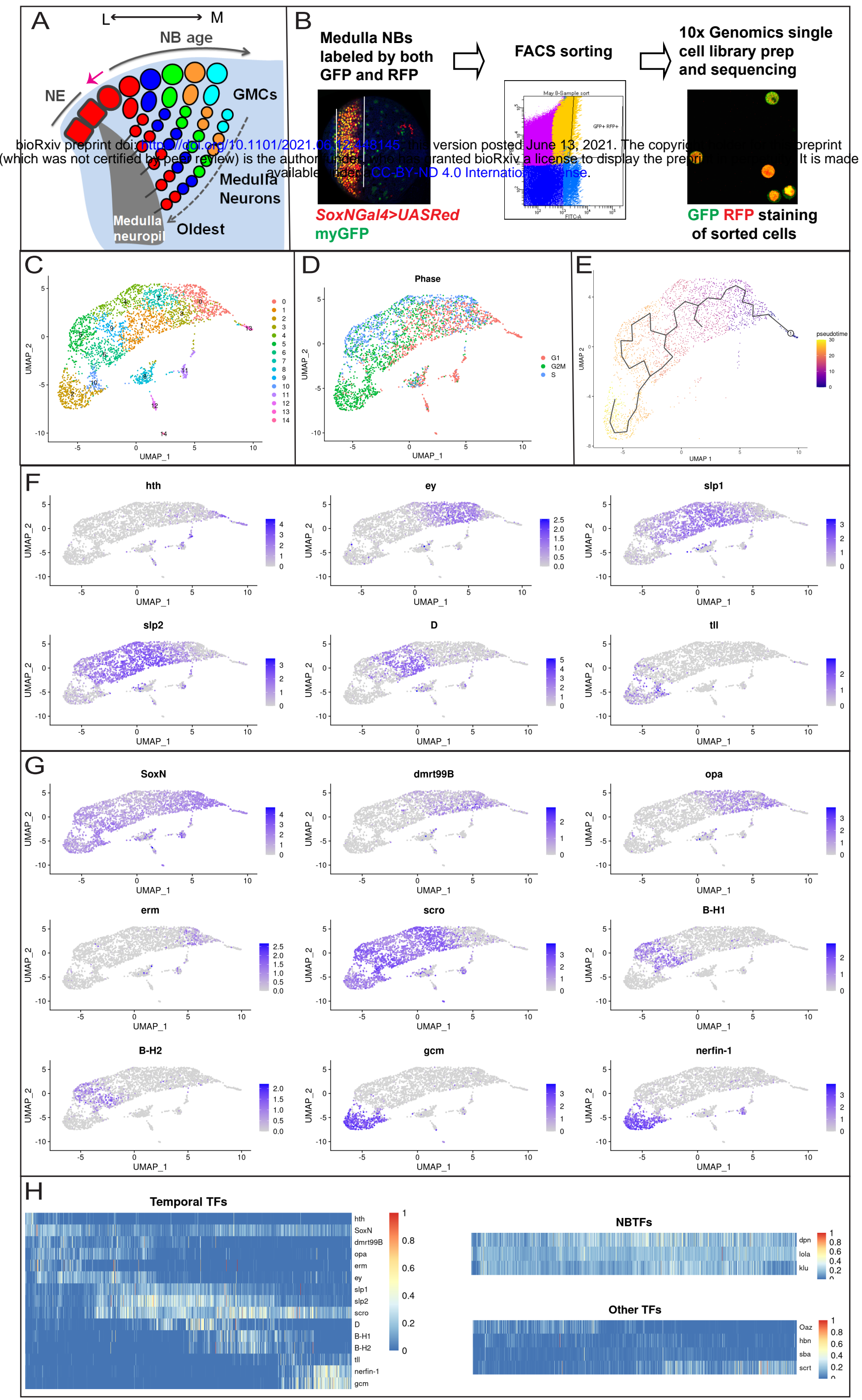


Figure 1. ScRNA-seq of Drosophila medulla neuroblasts. (A) A schematic drawing of the developing Drosophila medulla at the $3^{\text {rd }}$ instar larval stage. A neurogenesis wave (pink arrow) spreads from medial (M) to lateral (L), and sequentially converts NE cells into NBs. Thus, NBs from the youngest to the oldest are aligned on the lateral to medial axis. NBs sequentially express different TTFs, and generate differently fated progeny. The earliest-born neurons of each NB lineage are located closest to the medulla neuropil. The later-born neurons are located at more and more superficial layers. (B) The strategy and work-flow of the scRNA-seq of FACS sorted medulla NBs. Medulla NBs are uniquely labeled by the combination of SoxNGal4>UAS-RedStinger (red) and $E(s p l) m y G F P$ (green). Dissected larval brains were dissociated into single-cell suspension and subjected to FACS sorting to enrich medulla NBs. Then 10x V3 Single Cell libraries were generated using the sorted cells, and sequenced. (C) The sequenced cells were partitioned into 15 clusters using Seurat and visualized on UMAP plots. (D) The estimated cell cycle phase of each sequenced cell was visualized on UMAP plots. (E) Pseudotime trajectories generated using Monocle3, with the purple color stands for the earliest pseudotime, and yellow color stands for the latest pseudotime. (F) The expression patterns of known TTFs (Hth, Ey, SIp1, SIp2, D and TII) verify the pseudotime trajectories. (G) The expression patterns of newly identified TTFs visualized on UMAP plots. $(\mathrm{H})$ Heatmaps showing the expression levels of three classes of TFs across the pseudotime. Temporal TFs include known and novel TTFs; NBTFs include three TFs expressed in NBs of all ages; Other TFs include four TFs that show a temporal expression profile, but were not further characterized. The expression levels are visualized as a percentage of the maximum observed expression across all cells. 


\section{Figure 2}
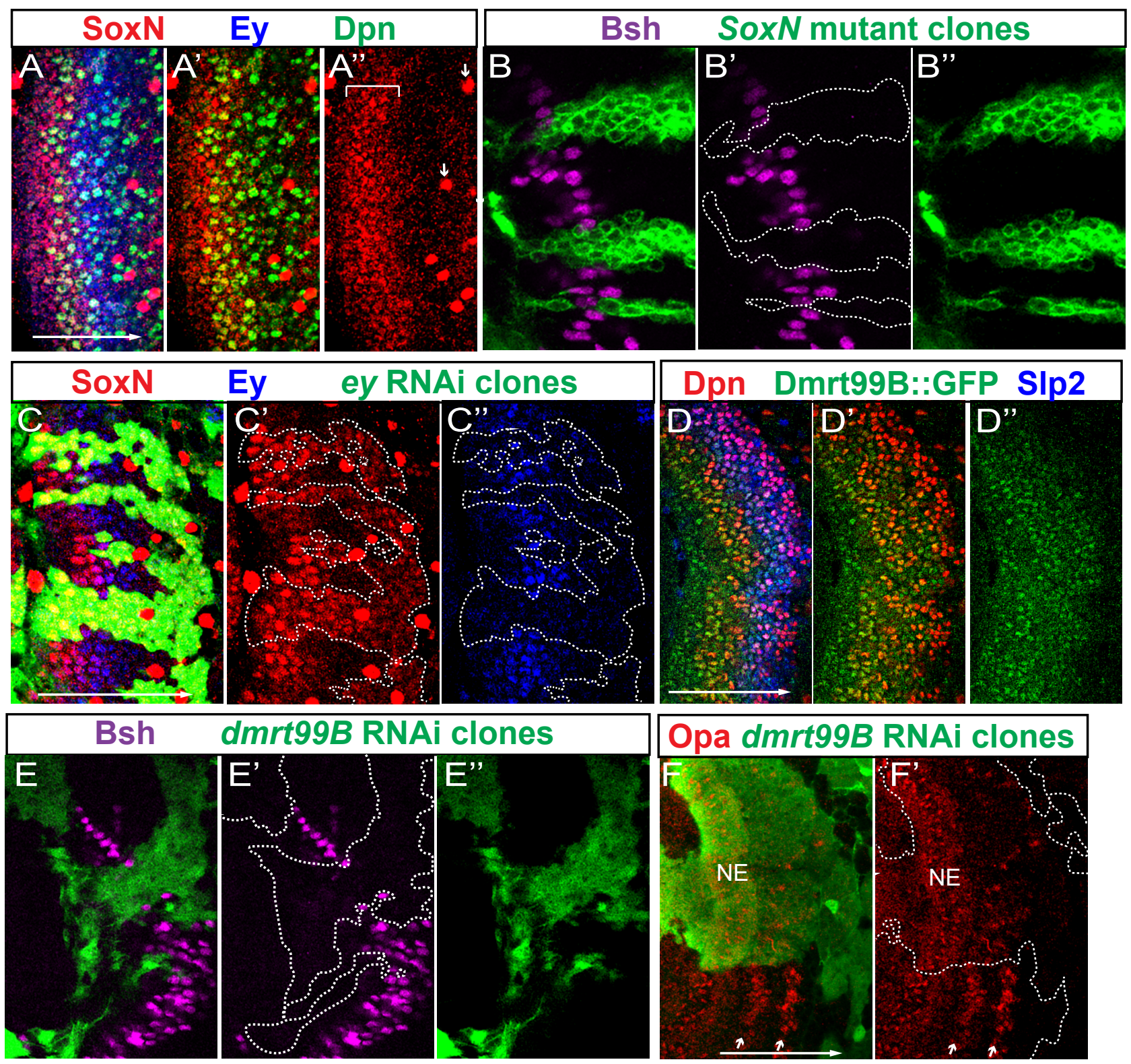

Figure 2. SoxN and Dmrt99B are two TTFs that function together with Hth in the first temporal stage. In all images of this and the following figures, lateral is to the left, and medial is to the right. The large white arrow from left to right indicates the NB age from the youngest to oldest. White dashed lines indicate clone margin unless otherwise noted. (A- $\left.A^{\prime \prime}\right)$ SoxN protein (red) is expressed before NB formation and in the youngest NBs (marked by Dpn in green) before Ey (blue), and this expression domain is indicated by a white bracket. SoxN is also expressed in glial cells, and examples are indicated by small white arrows. (B-B") In Sox $N^{N C 14}$ mutant clones marked by GFP (green), Bsh (magenta) is lost (in 12 out of 12 clones). (C-C') In eyRNAi clones marked by GFP (green), Ey (blue) is lost, and SoxN (red) expands into older NBs (in 11 out of 11 clones). (D-D") Dmrt99B::GFP (green) is expressed before NB formation and in the young NBs (NBs marked by Dpn in red) until early Slp (blue) stage. (E-E") In Dmrt99B-RNAi clones marked by GFP (green), Bsh (magenta) is lost (in 7 out of 7 clones). (F, $\mathrm{F}^{\prime}$ ) In Dmrt99B-RNAi clones marked by GFP (green), Opa (red) is lost or delayed (4 out of 4 clones). White arrows indicate the two stripes of Opa expression in NBs. 
Figure 3
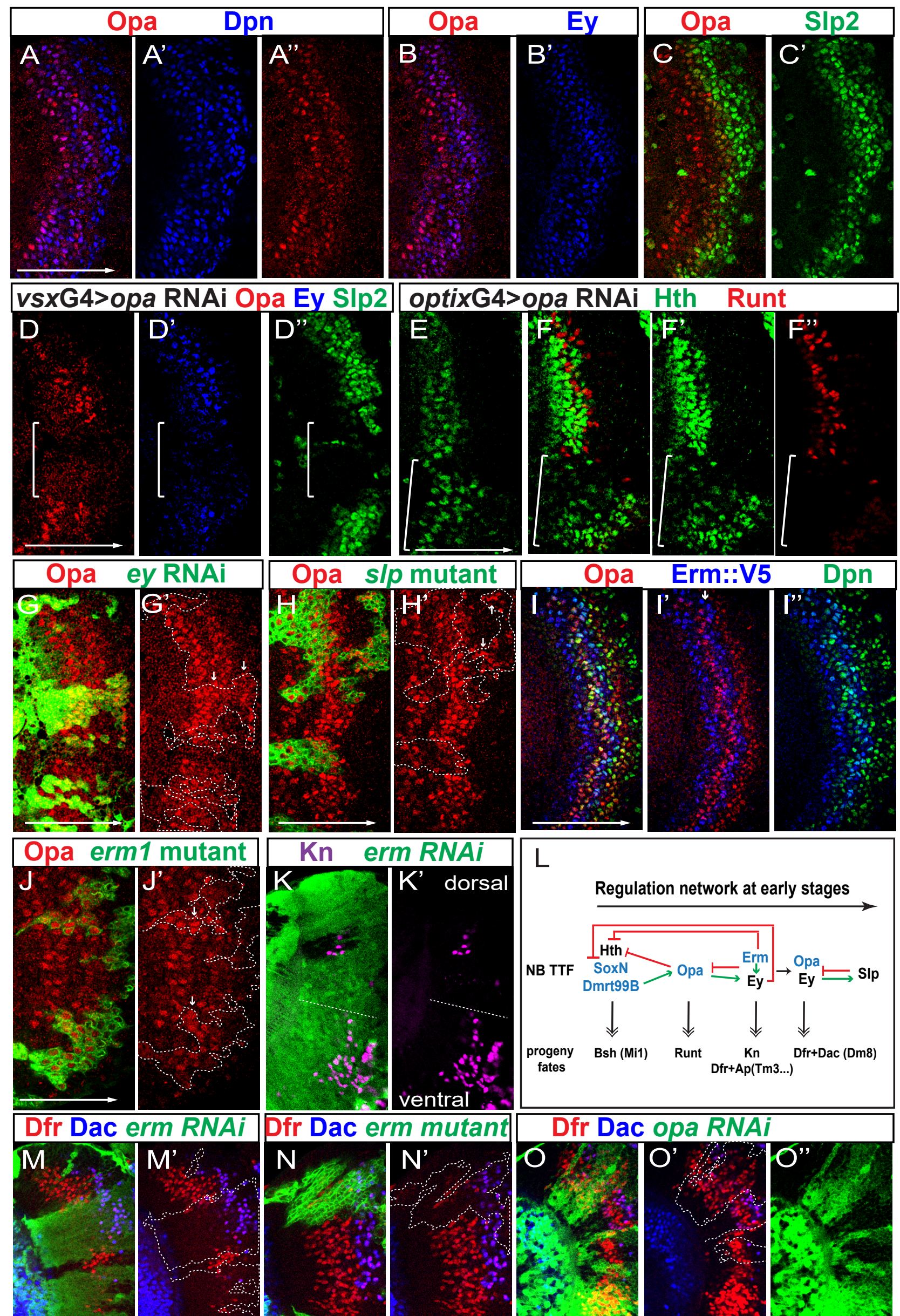
Figure 3. Opa and Erm are among the early TTFs. (A-A") Opa protein (red) is expressed in two stripes of NBs (marked by Dpn in blue). (B-B') The first stripe of Opa (red) is downregulated as Ey (blue) is upregulated, the second stripe of Opa overlaps with the Ey stripe. (C-C') The second stripe of Opa is downregulated as SIp (green) is upregulated. (D-D") When opaRNAi is driven by $v s x G a l 4$, Opa (red) is lost in the center domain (cOPC, white bracket), Ey (blue) and SIp2 (green) are also mostly lost in the same domain (in 11 out of 11 brains). (E-F") opaRNAi is driven by optixGal4 in mOPC indicated by a white bracket. (E) At a surface focal plane, Hth (green) is expanded into older NBs in mOPC (in 3 out of 3 brains). ( $\left.F-F^{\prime \prime}\right)$ At a deeper progeny focal plane, Hth expression (green) is expanded into later-born progeny, and Runt (red) expressing neurons are lost (in 3 out of 3 brains). (G, $G^{\prime}$ ) In ey-RNAi clones marked by GFP (green), Opa expression (red) is de-repressed in the gap and also expanded into older NBs (in 15 out of 15 clones). ( $H, H^{\prime}$ ) In s/p mutant clones marked by GFP (green), Opa expression (red) is expanded into older NBs (3 out of 3 clones). (I-I") Erm::V5 protein (blue) is expressed in two stripes, one before the NB (marked by Dpn in green) formation, and the other (white arrow) is between the two Opa stripes (red). (J,J') In erm ${ }^{1}$ mutant clones marked by GFP (green), Opa expression (red) is de-repressed in the gap (white arrows) (in 13 out of 13 clones). (K,K') In erm-RNAi clones marked by GFP (green), $\mathrm{Kn}$ (magenta) expressing neurons are lost on the dorsal side (in 15 out of 15 clones), but still present on the ventral side (in 13 out of 14 clones). A white dashed line separates the dorsal vs ventral side. (L) A schematic showing the regulatory network among early TTFs and the neuron fates generated at each stage. (M-M') In erm-RNAi clones marked by GFP (green), neurons expressing Dfr (red) but not Dac (blue) are lost, and the remaining neurons express both Dfr and Dac thus appear purple (in 13 out of 15 clones). ( $\left.N, N^{\prime}\right)$ In erm ${ }^{1}$ mutant clones marked by GFP (green), neurons expressing Dfr (red) but not Dac (blue) are lost, and the remaining neurons express both Dfr and Dac thus appear purple (in 11 out of 13 clones). (O-O') In opa-RNAi clones marked by GFP (green), neurons expressing both Dfr (red) and Dac (blue) are lost, but neurons expressing only Dfr are expanded (in 8 out of 8 clones). 
Figure 4

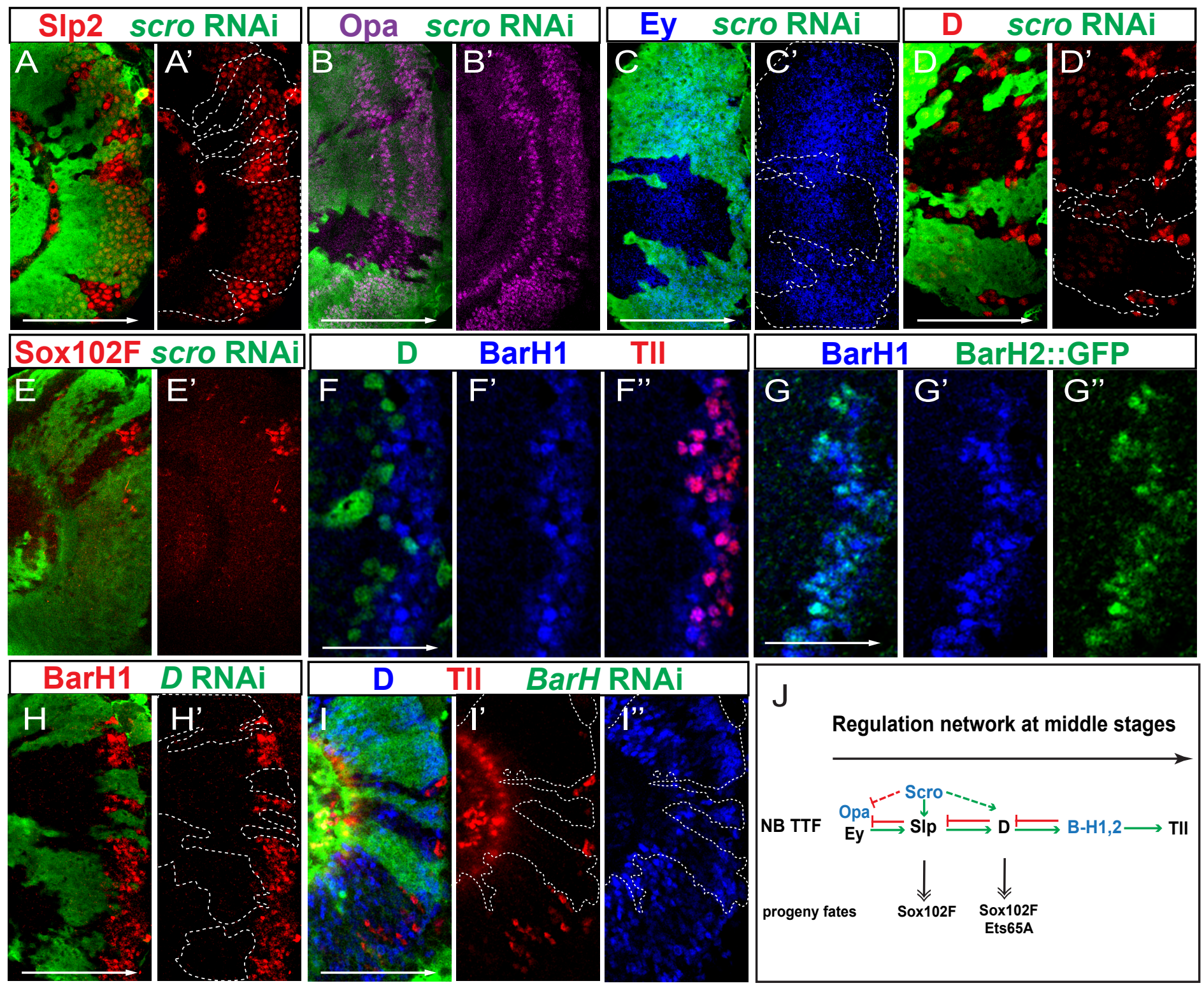

Figure 4. Scro and BarH proteins are among late TTFs. (A-A') In scro-RNAi (BDSC 33890) clones marked by GFP (green), Slp expression (red) is much weaker compared to outside of the clones (9 out of 9 clones). (B-B') In scro-RNAi (BDSC 33890) clones marked by GFP (green), Opa expression (magenta) is expanded into older NBs (8 out of 8 clones). (C-D') In scro-RNAi (BDSC 33890) clones marked by GFP (green), Ey expression (blue) is expanded into older NBs, and D expression (red) is lost ( 8 out of 8 clones). The $D$ antibody we used has unspecific cross-reactivity with other TFs in young neuroblasts (weak staining), but it specifically recognizes $D$ at the $D$ stage, which is demonstrated in Figure S2F. (E,E') In scro-RNAi (BDSC 29387) clones marked by GFP (green), Sox102F (red) expressing neurons are not generated (15 out of 17 clones show a complete loss). (F-F") The expression pattern of D (green), BarH1 (blue) and TIl (red) in NBs. (G$\left.\mathrm{G}^{\prime \prime}\right)$ The expression of BarH1 (blue) and BarH2::GFP (green) in NBs. ( $\mathrm{H}-\mathrm{H}^{\prime}$ ) In D-RNAi clones marked by GFP in green, BarH1 expression (red) is lost. (I-I') In BarH1 and BarH2 double RNAi clones marked by GFP (green), Tll expression (red) is lost, and D expression (blue) is expanded (in 15 out of 15 clones). (J) A schematic showing the regulatory network among late TTFs: Scro, Slp, $\mathrm{D}, \mathrm{BarH}, \mathrm{TII}$, and the neuron fates generated at each stage. 


\section{Figure 5}
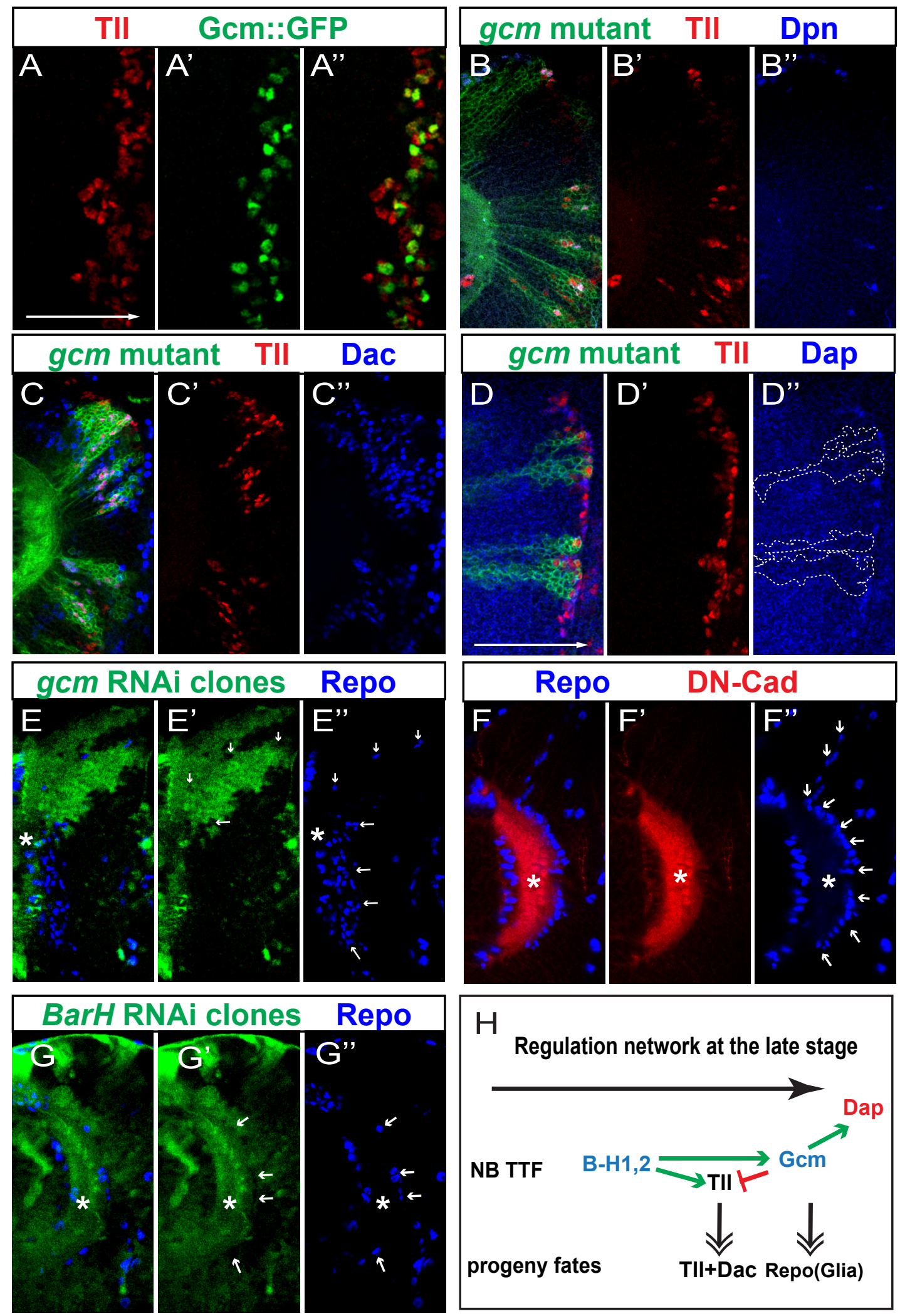
Figure 5. Gcm is the TTF that terminates the temporal cascade. (A-A") The expression of TII (red) and Gcm::GFP (green) in NBs. (B-B") In gcm mutant clones (marked by GFP in green), ectopic NBs marked by Dpn (blue) and TII (red) are present in a deep progeny focal plane, along with ectopic $\mathrm{TII}^{+}$progeny surrounding the ectopic NBs (in 17 out of 17 clones). (C-C') In gcm mutant clones (green), TIl (red) and Dac (blue) double positive cells are increased (in 15 out of 15 clones). (D-D") In $\mathrm{gcm}$ mutant clones (green), Dap (blue) is lost. The co-expression of TII (red) and Dap is only present outside the clones (in 6 out of 6 brains). (E-E') In gcm RNAi clones (marked by GFP in green), glia marked by Repo (blue) are mostly lost (in 12 out of 12 clones). The asterisk indicates the center of the neuropil, and the arrows are pointing at the glia. Note the glia don't have GFP expression. (F-F') In wild-type brains, mng marked by Repo (blue) migrate towards the neuropil marked by DN-Cad (red), and are aligned continuously around the neuropil surface. The asterisk indicates the center of the neuropil, and the arrows are pointing at the glia. (G-G') In BarH1 and BarH2 double RNAi clones marked by GFP (green), mng marked by Repo (blue) is greatly lost (in 6 out of 6 clones). The asterisk indicates the center of the neuropil, and the arrows are pointing at the glia. The remaining glia don't have GFP expression. $(\mathrm{H})$ A schematic showing the regulatory network of $\mathrm{Gcm}$ and its relating TTFs: BarH, TIl, and the progeny fates generated at the Gcm stage and the TIl stage. 
bioRxiv preprint doi: https://doi.org/10.1101/2021.06.12.448145; this version posted June 13, 2021. The copyright holder for this preprint (which was not certified by peer review) is the author/funder, who has granted bioRxiv a license to display the preprint in perpetuity. It is made available under aCC-BY-ND 4.0 International license.

\section{Figure 6}

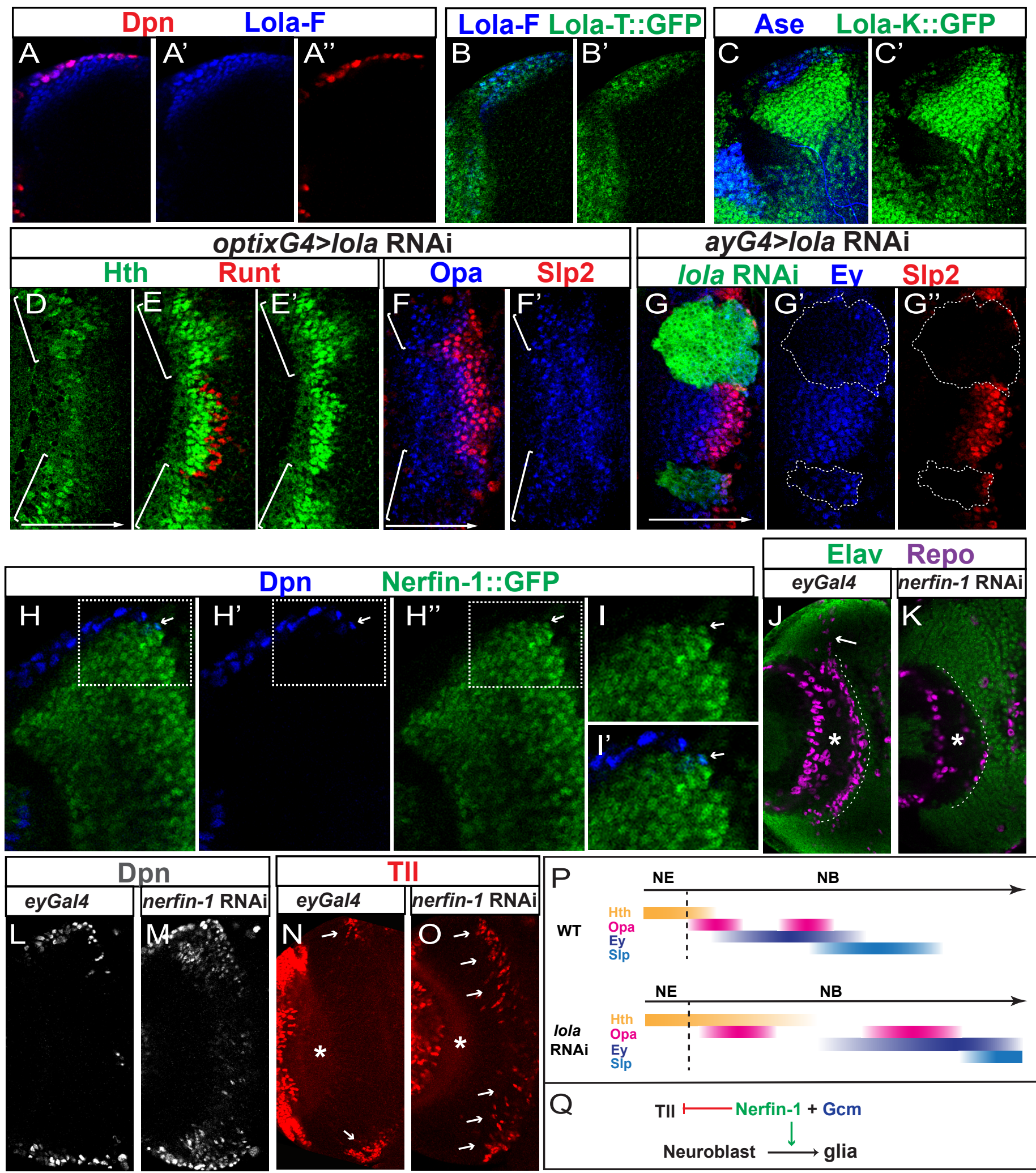


Figure 6. Lola and Nerfin-1 regulate the progression and termination of the temporal cascade, respectively. The lola RNAi line used in this figure is BDSC35721. (A-A") The expression of Dpn (red) and Lola-F (blue) in a cross-sectional view. (B,B') The expression of Lola-T::GFP (green) and Lola-F (blue) in a cross-sectional view. (C,C') The expression of Lola-K::GFP (green) and Ase (blue) that marks both NBs and GMCs in a cross-sectional view. (D-F') lola RNAi is driven by optixGal4 in $\mathrm{mOPC}$ indicated by white brackets. (D-E') With lola RNAi, Hth (green) is expanded into older NBs (D) and later-born progeny (E,E'), while Runt neurons (red) are mostly lost (E) (in 5 out of 5 brains). (F-F') With lola RNAi, the expression of the first stripe of Opa (blue) is slightly expanded, while the expression of the second stripe of Opa is greatly delayed (in 7 out of 7 brains). (G-G') In Iola RNAi clones, the expression of Ey (blue) and SIp2 (red) are greatly delayed (in 16 out of 16 clones). ( $\left.\mathrm{H}-\mathrm{H}^{\prime \prime}\right)$ The expression of Dpn (blue) and Nerfin-1::GFP (green) in a cross-sectional view. The right most NB is the oldest NB that turns on Nerfin-1 (arrow). (I-I') A zoomed-in image of the outlined region in panel $\mathrm{H}$. $(\mathrm{J}, \mathrm{K})$ In deep progeny focal planes, glia are marked by Repo (magenta), and neurons are marked by Elav (green). The asterisk indicates the center of the medulla neuropil, and the white dashed line indicates the position where mng should be aligned. $(\mathrm{J})$ In eyGal4 control brains $(n=5)$, mng continuously align the medulla neuropil, and the migrating mng stream is indicated by a white arrow. (K) When Nerfin-1 RNAi is driven by eyGal4, only scattered mng are observed (in 5 out of 5 brains). (L) At a focal plane slightly deeper than the surface focal plane, most NBs marked by Dpn in an eyGal4 control brain are located at the surface of the medulla. (M) When Nerfin-1 RNAi is driven by eyGal4, many ectopic NBs marked by Dpn are present inside the medulla (in 5 out of 5 brains). (N) At a deep progeny focal plane, $\mathrm{TII}^{+}$(red) progeny (white arrows) are only generated around the surface NBs, while no Tll+ cells are observed in the middle of the brain. (O) At a comparably deep progeny focal plane, when Nerfin-1 RNAi is driven by eyGal4, TII (red) expressing progeny continue to be produced throughout the brain (in 6 out of 6 brains). (P) A schematic showing the function of Lola in regulating the temporal cascade. Loss of Lola causes slowing down of the temporal progression. (Q) Nerfin-1 is required for the transition from the Tll stage to gliogenesis. 
Figure 7

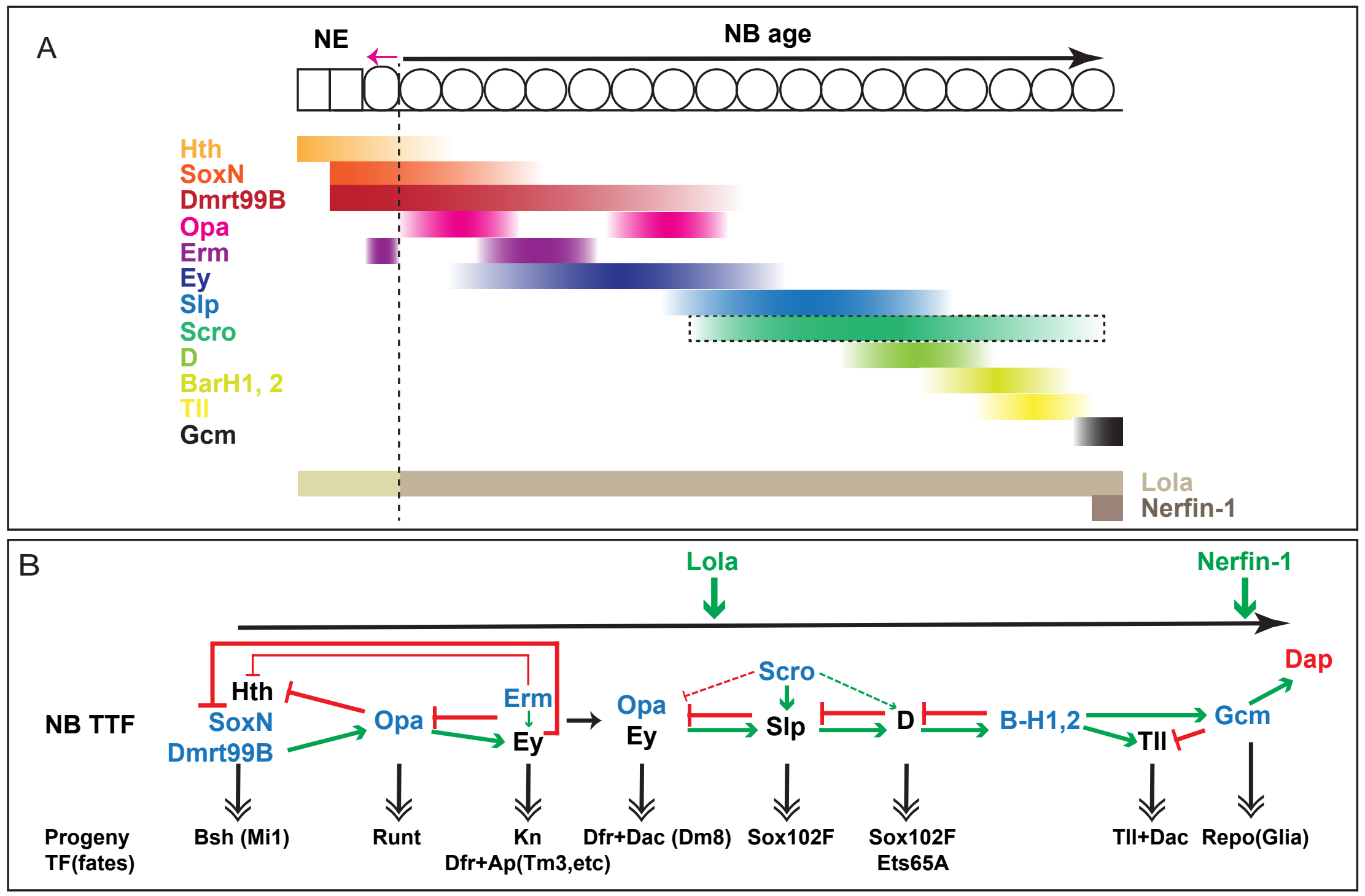


Figure 7. A schematic model summarizing the medulla TTF cascade and its regulation.

(A) A schematic drawing showing the relative expression patterns of the medulla TTFs, Lola and Nerfin-1. The expression of Scro is indicated only by its transcriptional pattern. The expression of Hth, SoxN and Dmrt99B all start in NE, Em has a stripe in the transition zone from NE to NB, while other TTFs are initiated in NBs. Different isoform compositions of Lola are indicated by different colors. The number of NE and NB cells do not indicate the actual number of cell cycles they go through. (B) A schematic model summarizing the regulation networks of the medulla TTF cascade. Known TTFs are in black, and new TTFs identified in this study are in blue. Extensive crossregulations were identified between these TTFs, that generally follow the rule that a TTF is required to activate the next TTF (green arrows) and repress the previous TTF (red flat-headed arrows), but with a few important exceptions. This TTF cascade controls the sequential generation of different neural types by regulating the expression of neuronal transcription factors, and examples of neural types were also indicated. Lola proteins modulate the speed of temporal progression of the NB TTF cascade. At the final stage, $\mathrm{Gcm}$ and Nerfin-1 promote gliogenesis and the cell-cycle exit to end the temporal progression. 


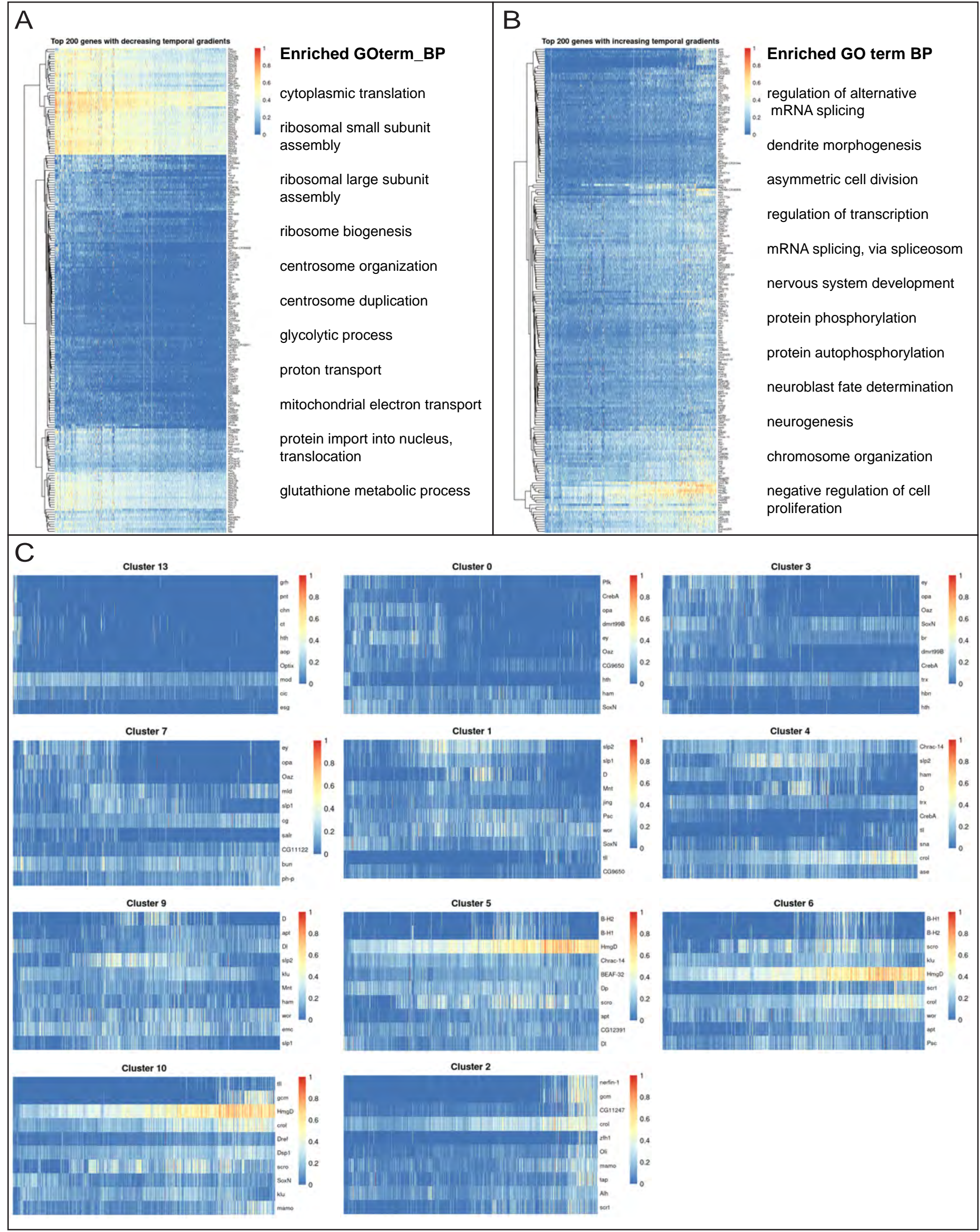


Supplementary Figure S1. Analysis of genes that show a temporal gradient of expression and TFs differentially expressed in each cluster. In all heatmaps, the expression levels are visualized as a percentage of the maximum observed expression across all cells. (A) Heatmaps showing the top 200 genes with decreasing temporal gradients across the pseudotime. Using the Functional Annotation Tool from the DAVID Bioinformatics Resources 6.8, these genes are enriched in the GO terms related to translation and metabolism. For details refer to Supplementary Table 2. (B) Heatmaps showing the top 200 genes with increasing temporal gradients across the pseudotime. These genes are enriched in the GO terms related to the regulation of gene expression, neural development, and signal transduction among others. For details refer to Supplementary Table 3. (C) Heatmaps showing the top 10 differentially expressed TFs for each NB cluster. The clusters are ordered according to their positions along the pseudotime. 


\section{Supplementary Figure S2}
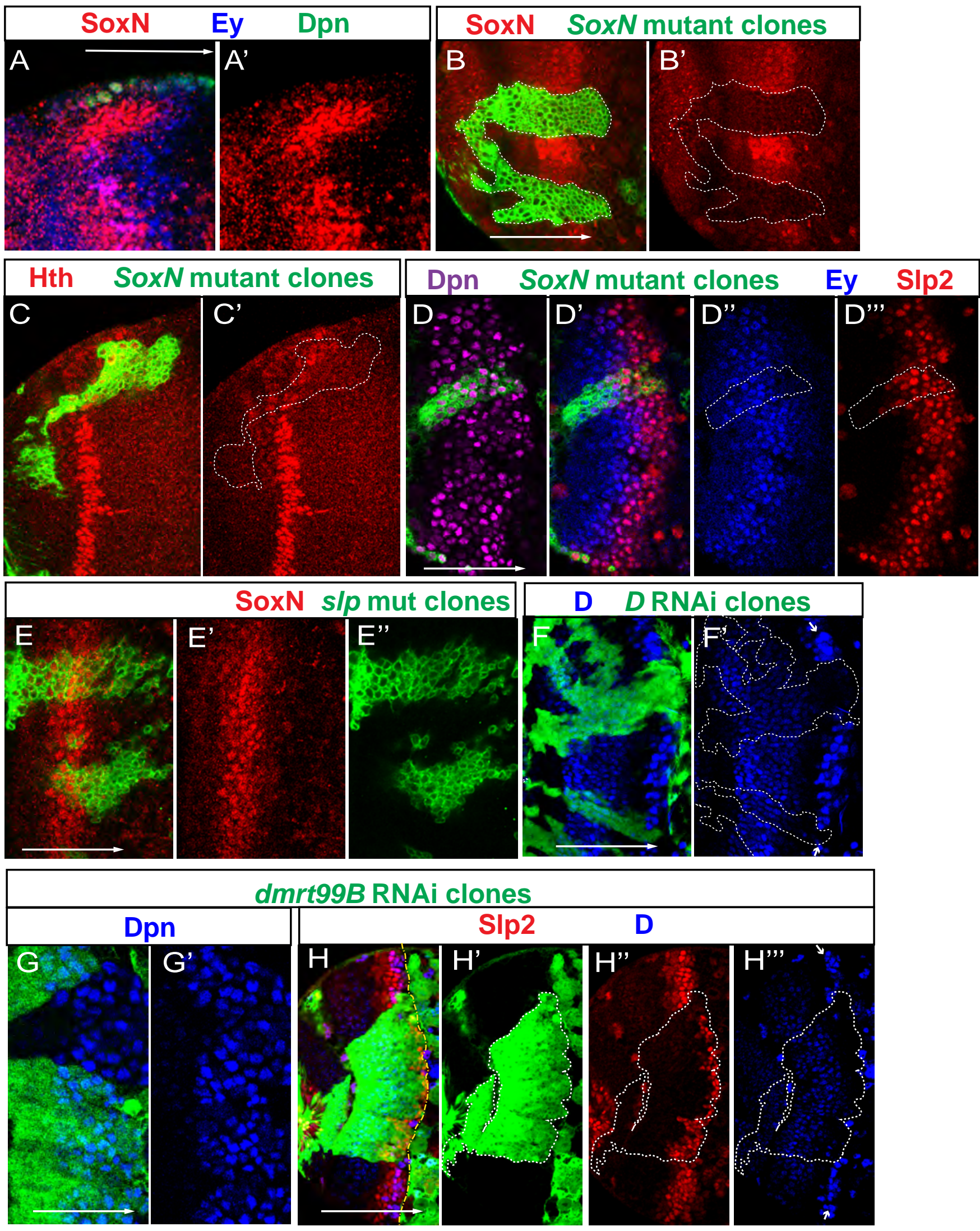
Supplementary Figure S2. Additional data for SoxN and Dmrt99B. (A-A") SoxN (red) is expressed in progeny generated before the Ey (blue) expressing progeny. NBs are marked by Dpn (green). $\left(B, B^{\prime}\right)$ In SoxN $N^{N C 14}$ mutant clones marked by GFP (green), SoxN (red) is lost (6 out of 6 clones). $\left(C, C^{\prime}\right)$ In SoxN $N^{N C 14}$ mutant clones marked by GFP (green), Hth expression (red) is normal (5 out of 5 clones). (D-D"') In SoxN ${ }^{N C 14}$ mutant clones marked by GFP (green), Ey (blue) and SIp2 (red) are still expressed in NBs marked by Dpn (magenta) (9 out of 9 clones). (E-E') In slp mutant clones marked by GFP (green), SoxN expression (red) is not affected ( 7 out of 7 clones). (F,F') The antibody for $D$ (blue) has unspecific cross-reactivity to other proteins in early NBs (the weak staining that is not lost in $D$-RNAi clones), while the second stripe of strong staining (white arrows) is specific for D, as the staining is lost in D RNAi clones (green). (G,G') In Dmrt99B-RNAi clones marked by GFP (green), NB formation is not affected as indicated by Dpn staining (blue) (11 out of 11 clones). (H-H'”') In Dmrt99B-RNAi clones marked by GFP (green), Slp expression (red) is delayed, and $D$ expression (strong blue staining indicated by white arrows) is lost (in 8 out of 8 clones). Note the weak blue staining in young NBs is cross-reactivity against other proteins. The white dashed lines in $\mathrm{H}^{\prime}-\mathrm{H}^{\prime \prime \prime}$ indicate clone margins, while the yellow dashed line with alternating short and long dashes in $\mathrm{H}$ indicates the boundary between the medulla and the central brain. 


\section{Supplementary Figure S3}
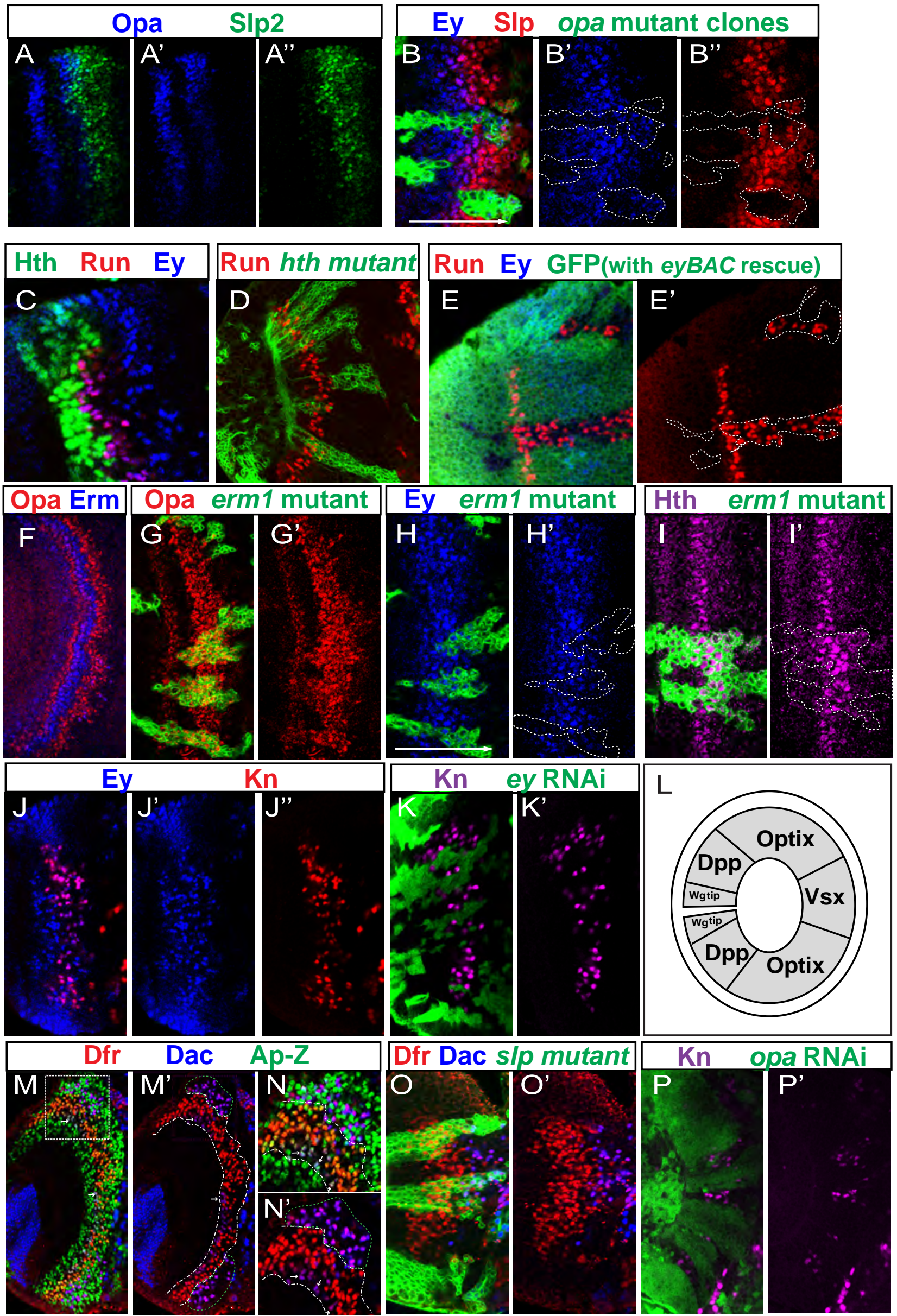
Supplementary Figure S3. Additional data for Opa and Erm. (A- $A^{\prime \prime}$ ) Opa (blue) is expressed in two layers of progeny, but is lost as neurons mature. Slp2 expression is in green. (B-B') In opa ${ }^{7}$ mutant clones (marked by GFP in green), the expression of Ey (blue) and SIp (red) is delayed (in 14 out of 14 clones). (C) Runt (red) expressing neurons are generated between the Hth (green) neurons and the Ey (blue) stage neurons. Note: Runt neurons later turn on Ey expression but they are not generated in the Ey stage. (D) In $h t h^{P 2}$ mutant clones marked by GFP (green), Runt (red) expressing neurons are still generated. (E, $\mathrm{E}^{\prime}$ ) In ey mutant clones marked by lack of GFP (green), Ey (blue) is lost, Runt (red) is expanded into later-born progeny. These negatively marked clones are generated in ey mutant background, and GFP positive cells contain the eyBAC rescue construct, while GFP negative cells are ey mutant (enclosed by white dashed lines). (F) Erm::V5 (blue) is expressed in a layer of progeny generated between the two layers of Opa (red) expressing progeny. $\left(\mathrm{G}, \mathrm{G}^{\prime}\right)$ In $\mathrm{erm}^{1}$ mutant clones marked by GFP (green) at a deeper progeny focal plane, the gap of Opa (red) expression in the progeny is also lost (in 13 out of 13 clones). $\left(\mathrm{H}, \mathrm{H}^{\prime}\right)$ In $\mathrm{erm}^{1}$ mutant clones marked by GFP (green) at the surface NB focal plane, Ey expression (blue) is still present but weaker (in 10 out of 10 clones). (I,I') In erm ${ }^{1}$ mutant clones marked by GFP (green), Hth expression (magenta) is slightly expanded (in 5 out of 5 clones). (J-J') Kn (red) is expressed in the Ey-expressing (blue) neurons generated by the Ey stage NBs. Note: A layer of earlier-born Ey expressing neurons (which are the Runt neurons that turn on Ey expression later), do not express $\mathrm{Kn}$. (K,K') In ey-RNAi clones marked by GFP (green), Kn (magenta) neurons are lost (in 10 out of 10 clones). (L) The schematic model of the compartmentalization of the medulla. $\left(\mathrm{M}-\mathrm{N}^{\prime}\right)$ The expression of Dfr (red), Dac (blue), and ap-LacZ (green) in neurons: a layer of earlierborn neurons express both Dfr and ap-LacZ (between the two white dashed lines in $\mathrm{M}^{\prime}-\mathrm{N}^{\prime}$ ), and several clusters of later-born neurons express both Dfr and Dac but not ap-LacZ (enclosed in green dashed circles). White arrows point to cells expressing Dfr, ap-LacZ and a weak level of Dac within the first population. $\left(\mathrm{N}, \mathrm{N}^{\prime}\right)$ A zoomed-in image of the outlined square in $\mathrm{M} .\left(\mathrm{O}, \mathrm{O}^{\prime}\right)$ In s/p mutant clones marked by GFP (green), Dfr (red) and Dac (blue) expressing neurons are still generated (10 out of 12 clones). (P, $\left.\mathrm{P}^{\prime}\right)$ In opa-RNAi clones marked by GFP (green), Kn (magenta) expression is lost (in 19 out of 20 clones). 


\section{Supplementary Figure S4}
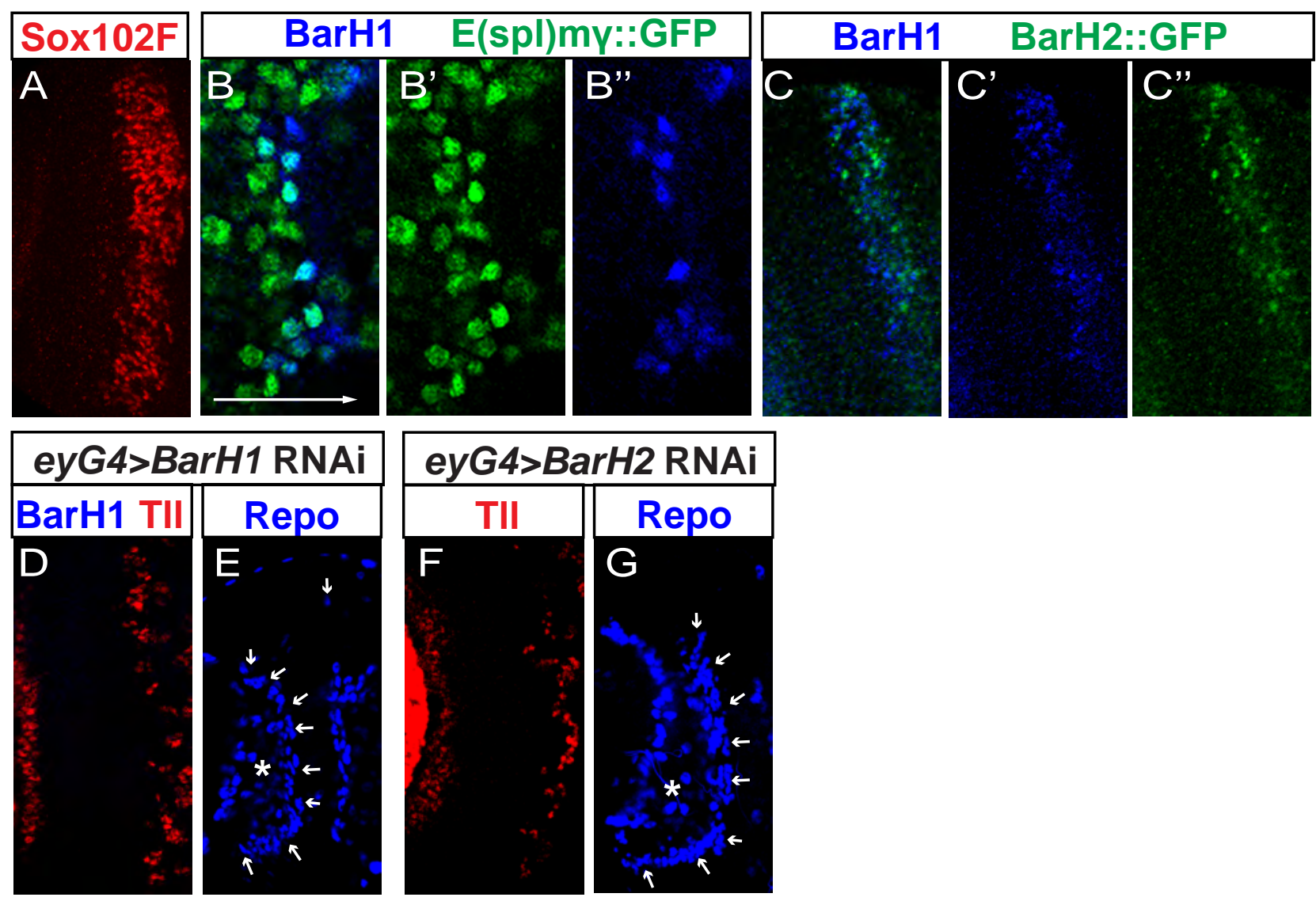

Supplementary Figure S4: Additional data for Scro and BarH. (A) The expression pattern of Sox102F (red) in neurons. (B-B") The expression of BarH1 (blue) in old medulla NBs marked by $\mathrm{E}(\mathrm{spl}) \mathrm{my}:: G F P$ (green). E(spl)my::GFP is expressed in all NBs. (C-C') The expression of BarH1 (blue) and BarH2::GFP(green) in neurons at a deep progeny focal plane. (D) At the surface NB focal plane, TIl (red) expression in NBs is not affected when BarH1 RNAi is driven by eyGal4 (in 4 out of 4 brains). (E) At a deep progeny focal plane, glia marked by Repo (blue) are not affected (in 4 out of 4 brains) when BarH1 RNAi is driven by eyGal4. The asterisk indicates the center of the neuropil and the arrows are pointing at the glia. (F) At the surface NB focal plane, TII (red) is not affected in NBs when BarH2 RNAi is driven by eyGal4 (in 3 out of 3 brains). (G) At a deep progeny focal plane, glia marked by Repo (blue) are not affected when BarH2 RNAi is driven by eyGal4 (in 3 out of 3 brains). The asterisk indicates the center of the neuropil, and the arrows are pointing at the glia. 


\section{Supplementary Figure S5}
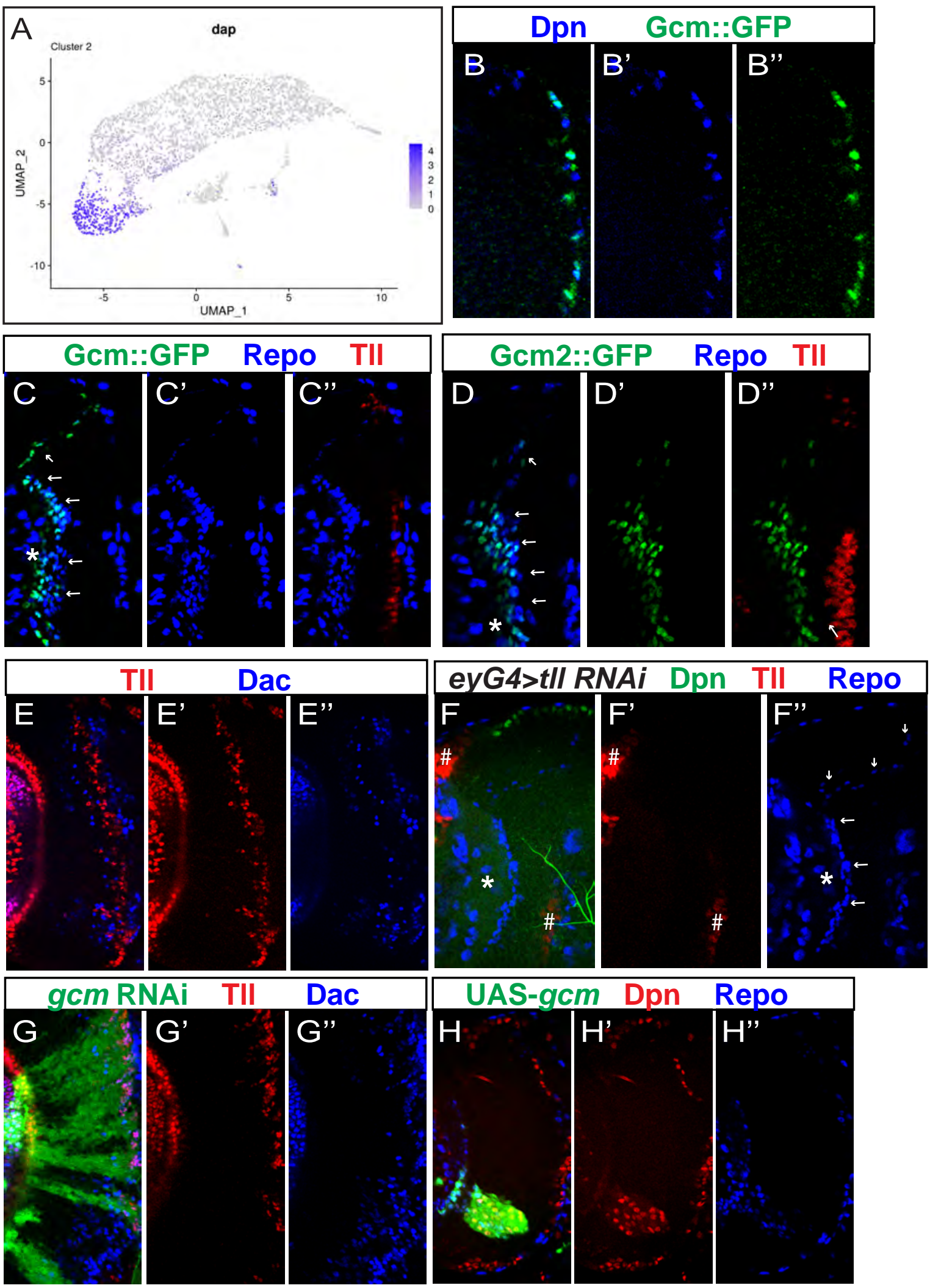
Supplementary Figure S5: Additional data for $\mathbf{G c m}$. (A) The mRNA expression pattern of Dap from the scRNA-seq data visualized on UMAP Plot. (B-B") The co-expression of Gcm::GFP (green) and Dpn (blue) in the oldest NBs, located between the medulla and the central brain, on a slightly deeper focal plane than younger NBs. (C-C') The expression of TIl (red), Gcm::GFP (green) and Repo (Blue) in a cross-sectional view. The asterisk indicates the center of the neuropil, and arrows point to mng. (D-D') The expression of TII (red), Gcm2::GFP (green) and Repo (blue) in a crosssectional view. The asterisk indicates the center of the neuropil, and arrows point to mng. (E-E') At a relatively superficial progeny focal plane close to the surface NBs, some TII (red) and Dac (blue) double positive neurons are present in the wild-type brain. (F-F") When t/l RNAi (tIl-miRNA) is driven by eyGal4, TII (red) is lost in NBs marked by Dpn (green), while glia marked by Repo (blue) are still aligned continuously around the medulla neuropil (in 3 out of 3 brains). The asterisk indicates the center of the neuropil, and arrows are pointing at the glia. The "\#" symbol indicates TIl expression in the NE of OPC and IPC is not affected because eyGal4 is not expressed there. (G$\mathrm{G}^{\prime \prime}$ ) In gcm RNAi clones (marked by GFP in green), TII (red) and Dac (blue) double positive cells are increased at a deep progeny focal plane (in 16 out of 16 clones). (H-H") In gcm misexpression clones (green), ectopic NBs marked by Dpn (red) are present, and ectopic glia marked by Repo (blue) are also present (in 2 out of 2 clones). 


\section{Supplementary Figure S6}
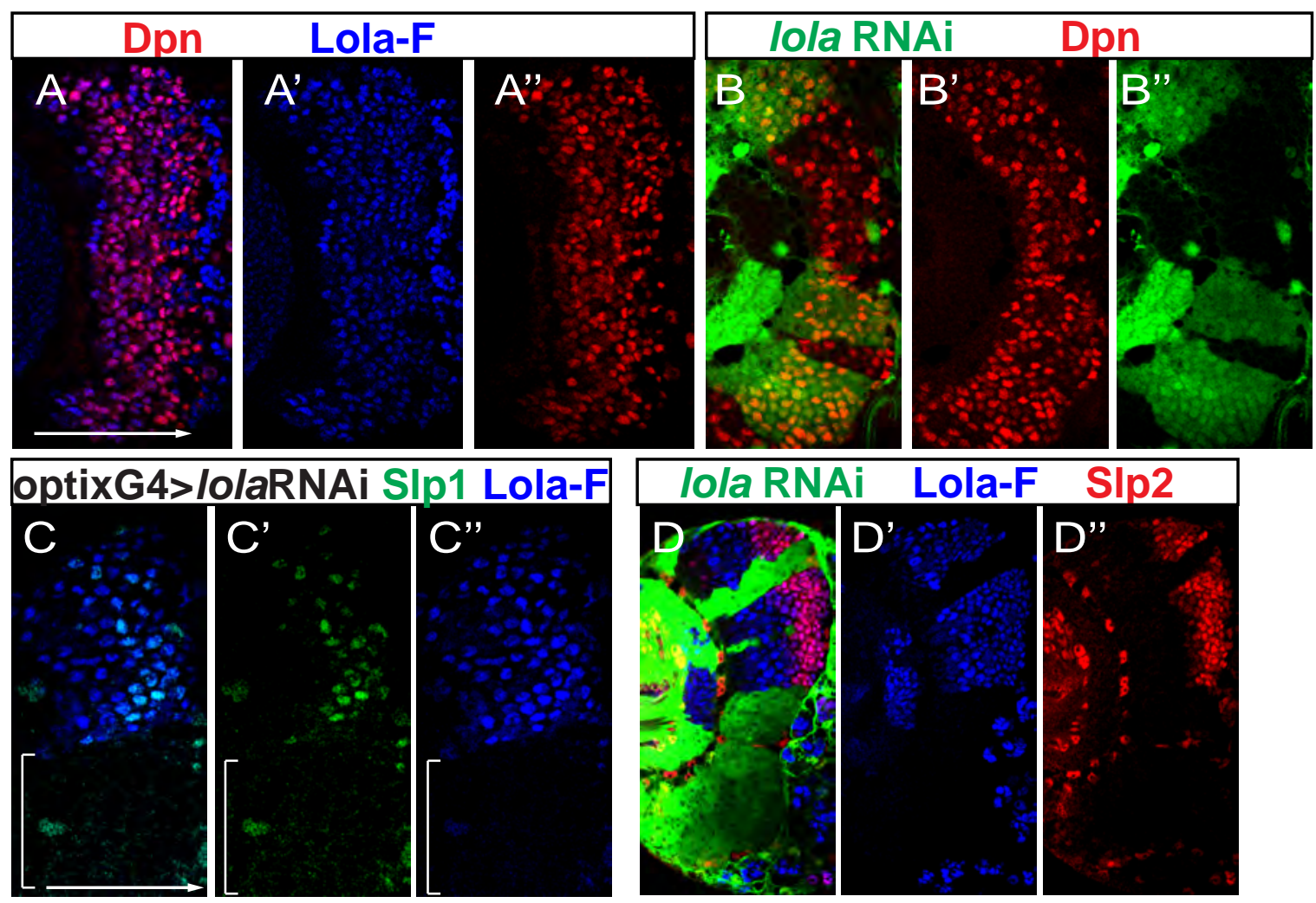

Supplementary Figure S6: Additional data for Lola and Nerfin-1. (A-A") The expression of Dpn (red) and Lola-F (blue) at a surface focal plane. (B-B") In lola RNAi (BDSC35721) clones marked by GFP in green, the formation of NBs (marked by Dpn in red) from NE cells is not affected. (C-C') When lola RNAi (BDSC35721) is driven by optixGal4, the expression of Lola (blue) is lost and the expression of SIp1 (green) is greatly delayed (in 4 out of 4 brains), in mOPC indicated by a white bracket. (D-D") In lola RNAi (VDRC 101925) clones marked by GFP (green), the expression of Lola (blue) is lost and the expression of Slp2 (red) is greatly delayed or lost (11 out of 11 clones). 
Supplementary Table 1. Known Drosophila cell cycle genes from Tinyatlas at Github (source: https://github.com/hbc/tinyatlas/blob/master/cell_cycle/Drosophila_melanogaster.csv).

This table includes known Drosophila cell cycle genes and the corresponding phase, and were used to estimate the cell cycle phase of each cell in our scRNA-seq data.

Supplementary Table 2. The top $\mathbf{2 0 0}$ genes showing decreasing gradients of expression and the enriched GOTERM analysis. Genes with temporally patterned expression gradients were identified as those whose expression levels showed significant Spearman correlation with the inferred pseudotime at an FDR cutoff of 0.05 . These genes were sorted according to the "corr" column (Spearman correlation), and top 200 genes with the highest negative values were analyzed for enriched Go terms for Biological Processes "GOTERM_BP_DIRECT" using the "Functional Annotation Chart" at the DAVID Bioinformatics Resources 6.8 website (https://david.ncifcrf.gov/home.jsp).

Supplementary Table 3. The top $\mathbf{2 0 0}$ genes showing increasing gradients of expression and the enriched GOTERM analysis. Genes with temporally patterned expression gradients were sorted according to the "corr" column (Spearman correlation), and top 200 genes with the highest positive values were analyzed for enriched Go terms for Biological Processes "GOTERM_BP_DIRECT" using the "Functional Annotation Chart" at the DAVID Bioinformatics Resources 6.8 website (https://david.ncifcrf.gov/home.jsp).

Supplementary Table 4. List of reagents used in this study with sources and Identifier numbers. 